US Army Corps of Engineers ${ }_{\circledast}$

Engineer Research and

Development Center

\title{
Developmental Design of the Remotely Piloted Vehicle System
}

Doris M. Turnage, Brent W. Towne, Burhman Q. Gates,

June 2019

Christopher L. Cummins, Robert D. Ellison, and Clint A. Barela

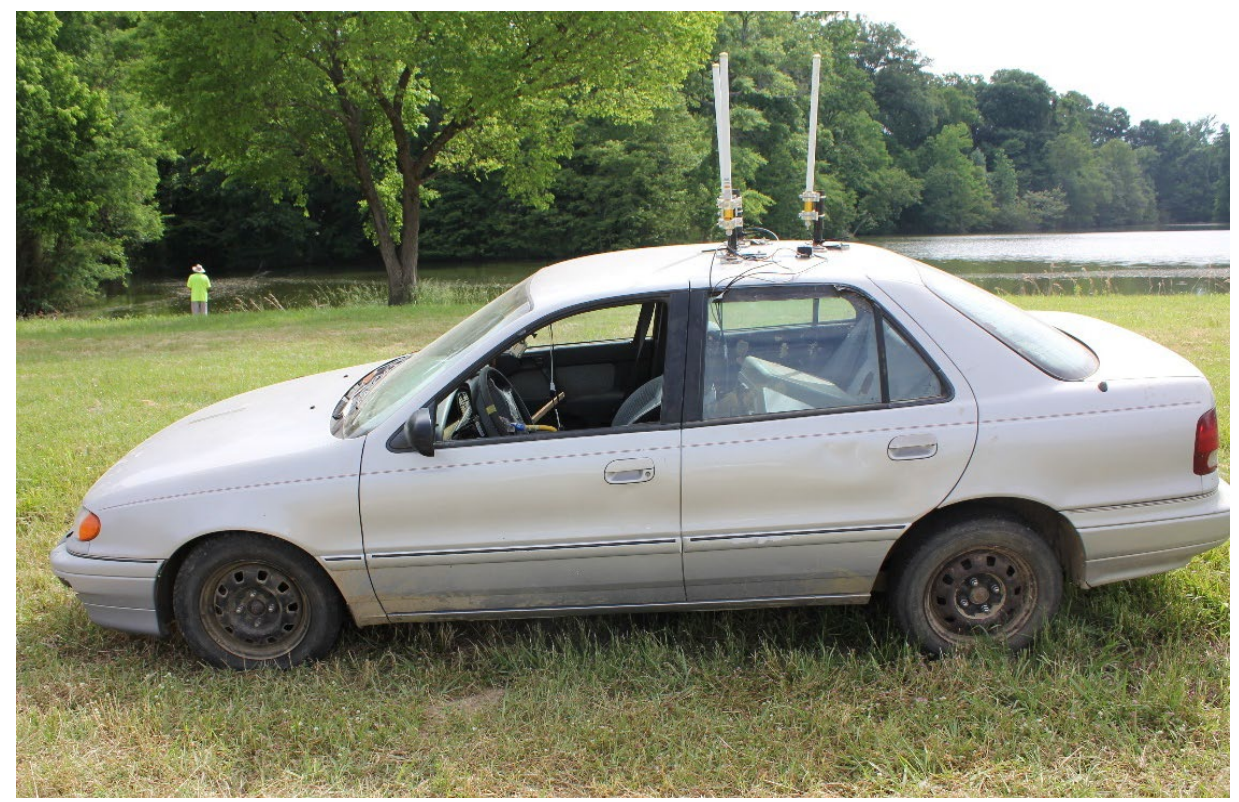


The U.S. Army Engineer Research and Development Center (ERDC) solves the nation's toughest engineering and environmental challenges. ERDC develops innovative solutions in civil and military engineering, geospatial sciences, water resources, and environmental sciences for the Army, the Department of Defense, civilian agencies, and our nation's public good. Find out more at www.erdc.usace.army.mil.

To search for other technical reports published by ERDC, visit the ERDC online library at http://acwc.sdp.sirsi.net/client/default. 


\section{Developmental Design of the Remotely Piloted Vehicle System}

Doris M. Turnage, Burhman Q. Gates, Christopher L. Cummins, Brent W. Towne, Robert D. Ellison, and Clint A. Barela

Geotechnical and Structures Laboratory

U.S. Army Engineer Research and Development Center 3909 Halls Ferry Road

Vicksburg, MS 39180-6918

Final report

Approved for public release; distribution is unlimited.

Prepared for U.S. Army Corps of Engineers

Washington DC 20314-1000

Under Project Number 455651 


\section{Abstract}

The U.S. Army Engineer Research and Development Center is developing a remotely piloted vehicle driving system for vehicles used in characterizing the performance of barrier systems against vehicle ramming attacks. The design of the driving system allows for expedient installation into a wide range of consumer vehicles with automatic transmissions.

Several successful vehicle ramming experiments were conducted using the remotely piloted vehicle ramming system. The design of the experiments allowed the user to characterize the performance of a barrier wall and an entry-point control system. The driver system successfully operated in sedans, trucks, and full-sized vans at target speeds varying from 30 to $60 \mathrm{mph}$. The vehicles operated safely at distances up to $1,500 \mathrm{ft}$ from the remote base station. The portability of the remotely piloted driver system allows for use at multiple test facilities.

This report documents features of the vehicle system and the pilot control system. These features include the vehicle dashboard camera that transmits video to the remote base station, cruise control that operates the vehicle at a target speed, shock sensors that shut down the vehicle within seconds after impact, emergency safety considerations, acceleration, and global positioning system (GPS) instrumentation measured on vehicle, and portability for incorporation into almost any vehicle.

DISCLAIMER: The contents of this report are not to be used for advertising, publication, or promotional purposes. Citation of trade names does not constitute an official endorsement or approval of the use of such commercial products. All product names and trademarks cited are the property of their respective owners. The findings of this report are not to be construed as an official Department of the Army position unless so designated by other authorized documents. 


\section{Contents}

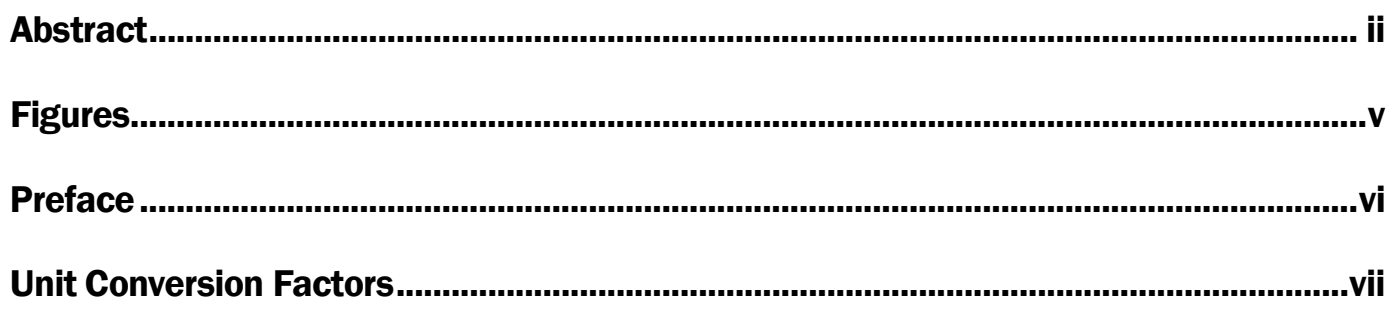

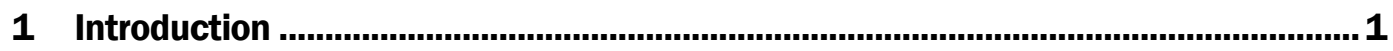

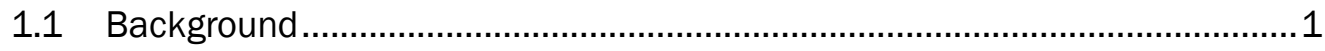

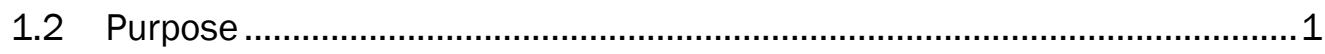

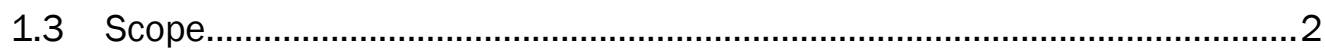

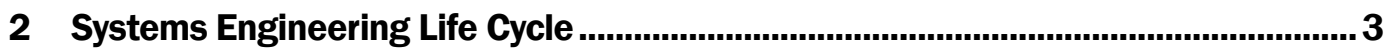

3 System of Systems Design................................................................................10

3.1 Vehicle system ................................................................................. 11

3.1.1 Vehicle driver component ........................................................................... 11

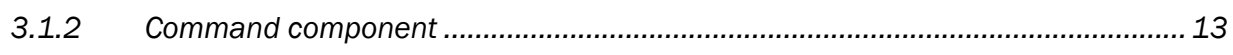

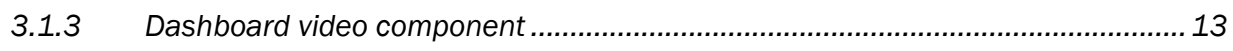

3.1.4 Fail-safe component ............................................................................................ 14

3.1.5 Vehicle state components ............................................................................... 15

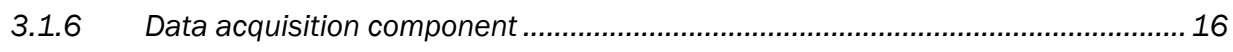

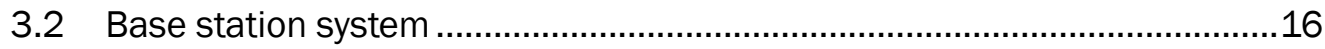

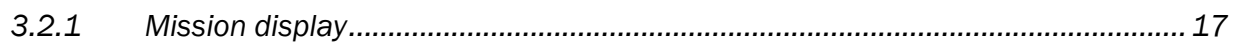

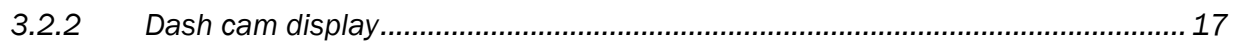

3.2.3 Vehicle state display .................................................................................. 18

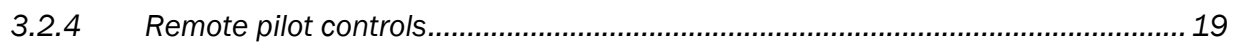

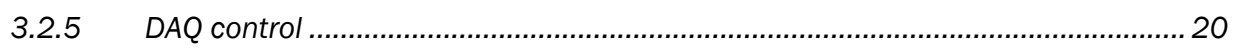

3.3 Test site .................................................................................... 20

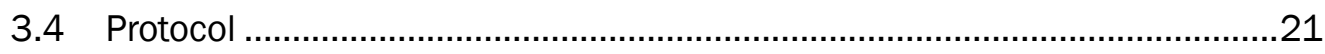

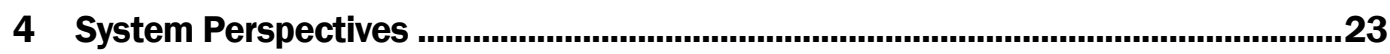

4.1 Driver perspective system .................................................................. 23

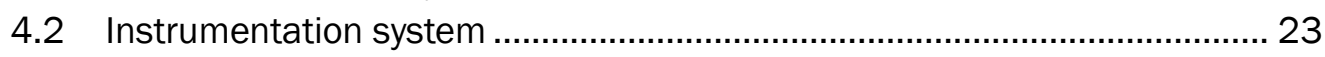

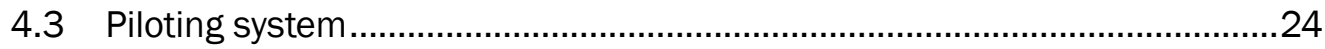

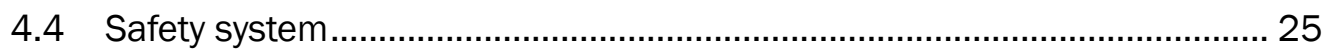

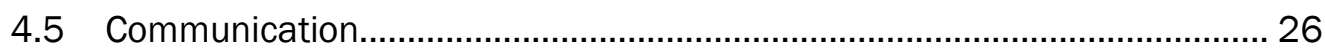

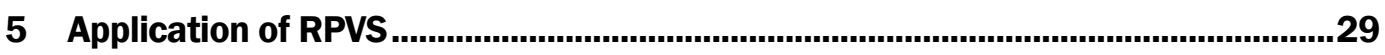

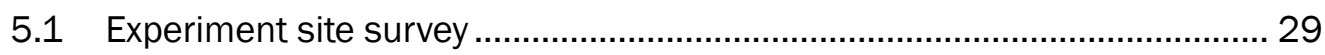

5.2 Experiment site setup............................................................................ 29

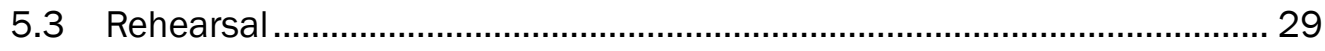

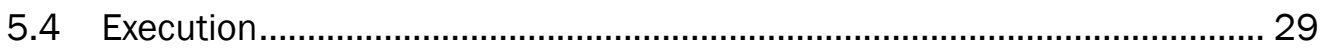




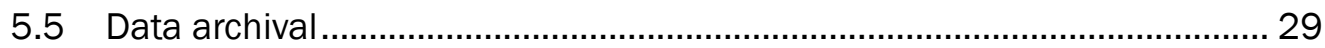

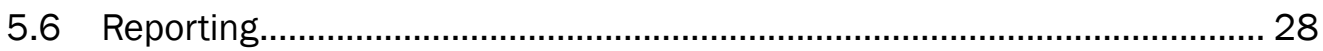

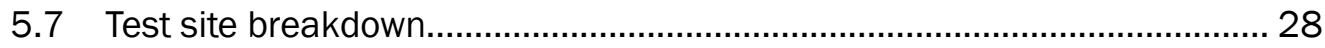

6 Performance Metrics.......................................................................................29

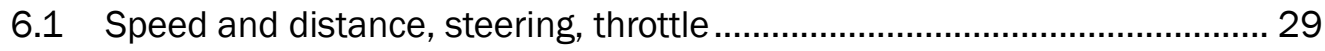

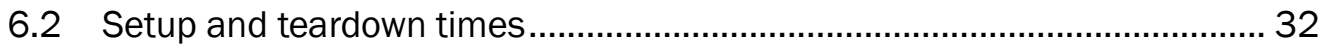

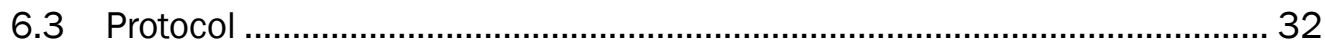

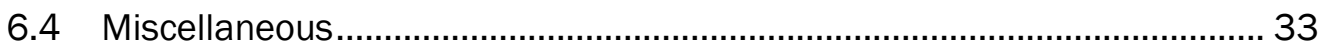

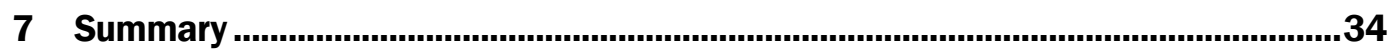

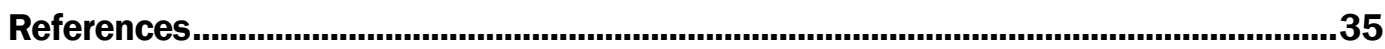

Appendix A: Issues....................................................................................................36

Appendix B: Materials List.......................................................................................37

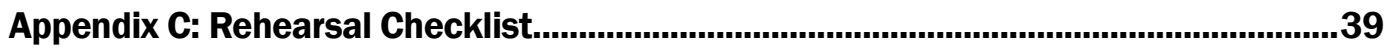

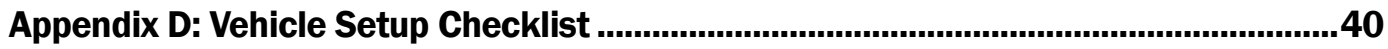

Report Documentation Page 


\section{Figures}

Figure 1. The system development life cycle. .................................................................. 4

Figure 2. Remotely piloted vehicle system. ......................................................................10

Figure 3. Example of vehicle with remotely piloted vehicle system installed......................11

Figure 4. Braking, throttle, and steering actuator assembly............................................12

Figure 5. Diagram of actuator assembly connected to vehicle.........................................13

Figure 6. Dashboard camera. ....................................................................................14

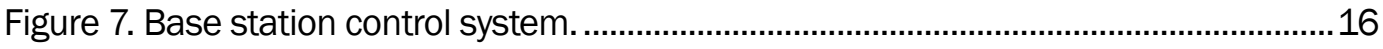

Figure 8. Mission display. ........................................................................................... 17

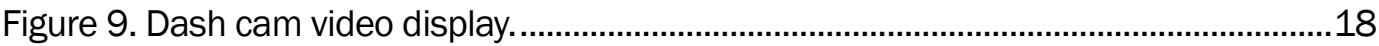

Figure 10. Vehicle state display. ..............................................................................19

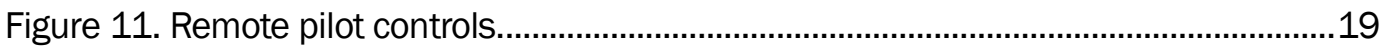

Figure 12. Test site includes base station, vehicle, people, and communication

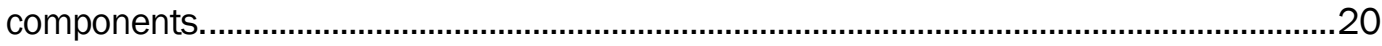

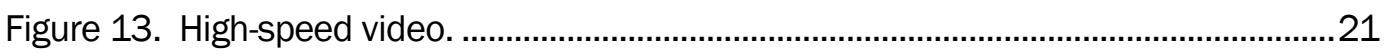

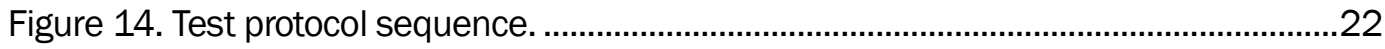

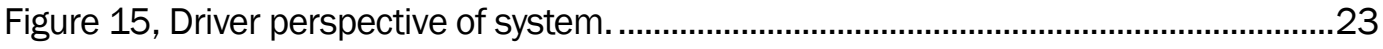

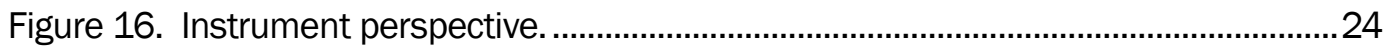

Figure 17. Piloting system perspective.......................................................................25

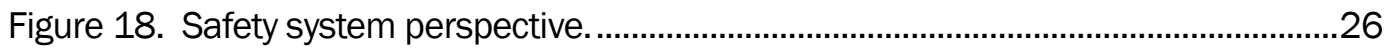

Figure 19. Communication perspective of system.............................................................2

Figure 20. Remotely piloted vehicle system data (11/12/2015 test).............................30

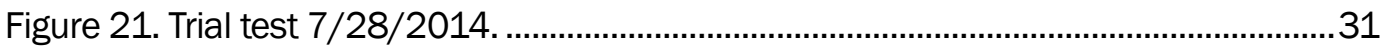

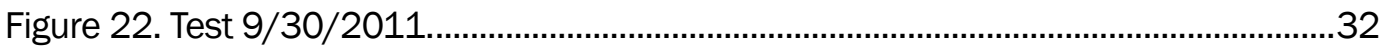




\section{Preface}

This work was conducted for the Deployable Force Protection and Force Protection Basing programs, under Project Number 455651.

This work was performed by the Mobility Systems Branch (MSB) of the Engineering Systems and Materials Division (ESMD), U.S. Army Engineer Research and Development Center, Geotechnical and Structures Laboratory (ERDC-GSL). At the time of publication, Dr. Jeff Durst was Chief, MSB; Mr. Jeffrey G. Averett was Acting Chief, ESMD; and Ms. Pamela G. Kinnebrew was the Technical Director for Military Engineering. The Deputy Director of ERDC-GSL was Mr. Charles W. Ertle, and the Director was Mr. Bartley P. Durst.

COL Ivan P. Beckman was the Commander of ERDC, and Dr. David W. Pittman was the Director. 


\section{Unit Conversion Factors}

\begin{tabular}{|l|c|l|}
\hline Multiply & \multicolumn{2}{l|l|}{ lo Obtain } \\
\hline feet & 0.3048 & meters \\
\hline foot-pounds force & 1.355818 & joules \\
\hline gallons (U.S. liquid) & $3.785412 \mathrm{E}-03$ & cubic meters \\
\hline hectares & $1.0 \mathrm{E}+04$ & square meters \\
\hline horsepower (550 foot-pounds force per second) & 745.6999 & watts \\
\hline inches & 0.0254 & meters \\
\hline inch-pounds (force) & 0.1129848 & newton meters \\
\hline kilotons (nuclear equivalent of TNT) & 4.184 & terajoules \\
\hline knots & 0.5144444 & meters per second \\
\hline ounces (mass) & 0.02834952 & kilograms \\
\hline ounces (U.S. fluid) & $2.957353 \mathrm{E}-05$ & cubic meters \\
\hline pints (U.S. liquid) & $4.73176 \mathrm{E}-04$ & cubic meters \\
\hline pints (U.S. liquid) & 0.473176 & liters \\
\hline tons (force) & $8,896.443$ & newtons \\
\hline tons (force) per square foot & 95.76052 & kilopascals \\
\hline tons (long) per cubic yard & $1,328.939$ & kilograms per cubic meter \\
\hline tons (nuclear equivalent of TNT) & $4.184 \mathrm{E}+09$ & joules \\
\hline tons (2,000 pounds, mass) & 907.1847 & kilograms \\
\hline tons (2,000 pounds, mass) per square foot & $9,764.856$ & kilograms per square meter \\
\hline yards & 0.9144 & meters \\
\hline
\end{tabular}


This page intentionally blank. 


\section{Introduction}

\subsection{Background}

The U.S. Army Engineer Research and Development Center (ERDC) has ongoing research requiring vehicle ramming and the resulting performance of vehicle-arrest barriers. The vehicle-arrest barriers were in development as non-lethal control measures at base entry points. A system to remotely pilot vehicles was initially developed to meet barrier evaluation and safety requirements. However, as additional barrier evaluations were conducted, the remote piloting system went through an evolution that met evolving design goals such as ease and speed of installation, setup, and teardown, robustness for field testing, and maintenance. During this evolution, the integration of a collection of distinct systems progressed to meet all design goals. The remotely piloted vehicle system (RPVS) is in development by the ERDC Mobility Systems Branch and used in conjunction with other Geotechnical and Structures Laboratory researchers for conducting crash evaluations of vehicle barrier systems.

Past research with the RPVS supported the Deployable Force Protection (DFP) Project and the Force Protection Basing. ERDC's Survivability Engineering Branch played an integral role in the DFP Science and Technology (S\&T) initiative since its inception and serves as the lead organization for the initiative, along with other U.S. Army, Air Force, and Navy organizations. The DFP program seeks to develop capabilities that provide additional protection for soldiers in small forward deployed bases while reducing time and logistic requirements.

Several successful vehicle ramming experiments were conducted using the RPVS. These experiments characterized the performance of a barrier wall and an entry-point control system. The portable remotely piloted driver system has successfully operated small sedans and trucks at speeds above $40 \mathrm{mph}$ and at distances up to $1,500 \mathrm{ft}$ from the remote base station.

\subsection{Purpose}

The purpose of this report is to document the evolution and high-level design of the RPVS, its components, the processes, and parts that comprise the system of systems. The high-level design displays the 
relationship between the vehicle systems, base station system, and the communication system.

\subsection{Scope}

This report provides an overview of the RPVS. Chapter 2 provides an overview of the systems engineering life cycle. Chapter 3 provides details on the development of the RPVS and it functionality. Chapter 4 provides details of various perspectives of the RPVS. Chapter 5 provides details on actual test preparations on the day of a test, along with the personnel, procedures, and equipment requirements. Chapter 6 provides various charts displaying throttle and braking information collected during various tests. Chapter 7 provides a summary of this report. 


\section{Systems Engineering Life Cycle}

Systems engineering (SE) is an interdisciplinary approach and means to enable the realization of successful systems (Department of Defense Systems Management College. 2001). Successful systems must satisfy the needs of their customers, users, and other stakeholders. In the broad community, the term "system" may mean a collection of technical, natural, or social elements or a combination of all three (http://sebokwiki.org/wiki/Systems_Engineering_Overview, accessed June 8, 2016).

The systems development life cycle (SDLC) is a period of time that begins from the time the idea is conceived or a problem identified and ends at the time the system is no longer useful or ended. The intermediate phase varies depending on the chosen SDLC model.

An SDLC should result in a system that meets or exceeds customer expectations, within time and cost estimates, works effectively and efficiently in the current and planned information technology infrastructure, and is inexpensive to maintain and cost-effective to enhance.

The systems engineering process (SEP) is a comprehensive, iterative, and recursive problem-solving process that is applied sequentially top-down by integrated teams.

Figure 1 shows the SDLC for the RPVS. It contains the following stages, (1) system initiation, (2) system requirements analysis, (3) system design stage, (4) system construction, (5) system testing, (6) system maintenance, and (7) system retirement. Discussion of each of these stages follows. The system stages 2 through 6 could be revisited at any time if the need arises to improve, repair, or update any of the system components. 
Figure 1. The system development life cycle.

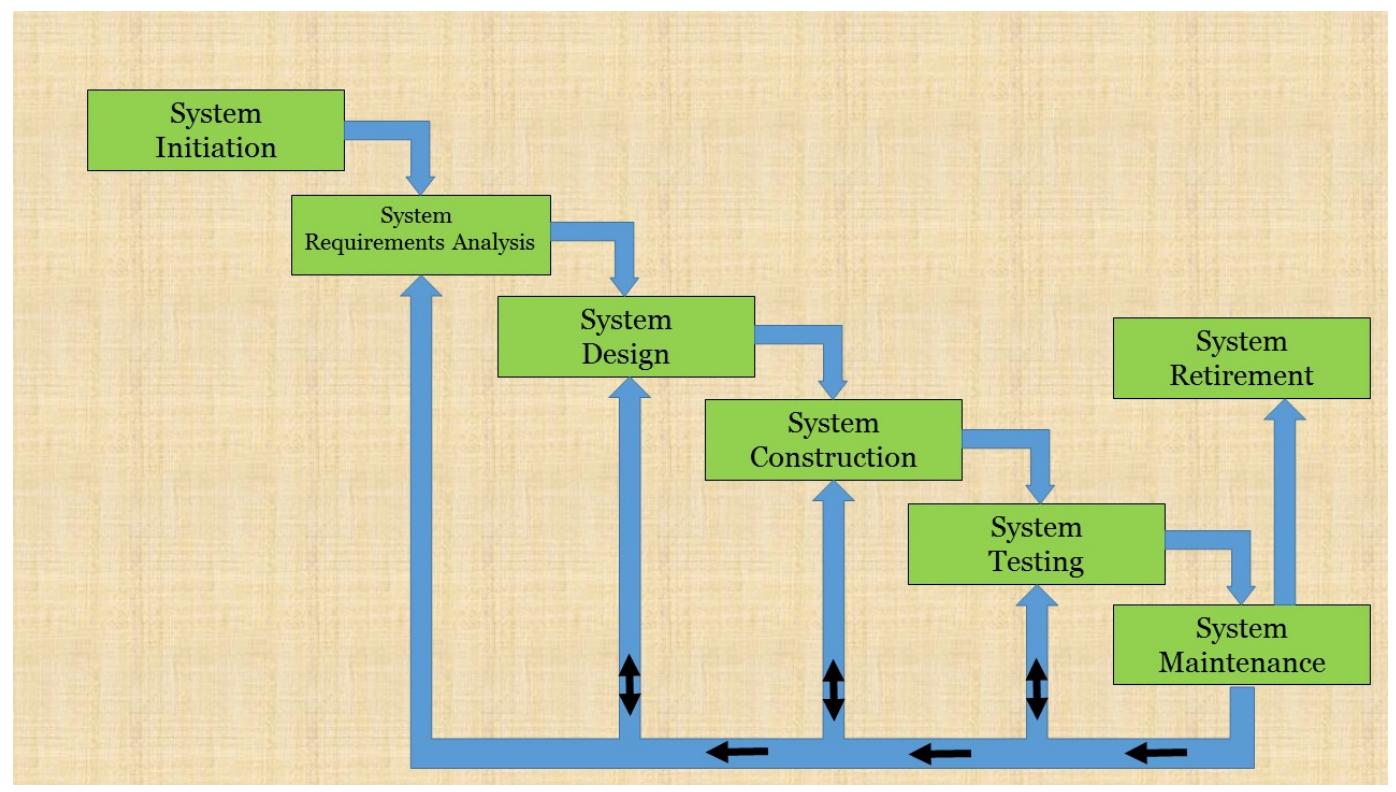

The System Initiation Stage begins when a need or opportunity arises for a system. The original problem for the RPVS identified and requested a methodology to conduct a crash test to measure barrier performance. The original request lacked explicit details for testing. The team relied on the details provided by the American Society for Testing and Materials (ASTM) standard for barrier crash testing. While previous testing used a remotely piloting system, no publications existed to show the requirements of that system and its evolution. The RPVS System Initiation stage was based on a need for a remotely piloted vehicle system to conduct two barrier system tests with the possibility of a third test. Requirements were then set during the System Requirements Analysis stage. The Analysis stage decomposes the systems requirements into smaller chunks in order to develop an actual product. Listed as follows are the initial requirements of the RPVS.

- Conduct barrier test according to ASTM specifications at a 40-mph level. ASTM barrier testing requirements included the following.

- Impact speed of 37 to $48 \mathrm{mph}$

- Vehicle must have stock bumper, no damage or reinforcement

- Onboard accelerometers located at the vehicle's center of gravity (CG) and optional second accelerometers located at center above rear axle.

- High-speed camera located perpendicular to barrier

○ Vehicle in free roll state after impact 
- Post impact area of $98 \mathrm{ft}$.

- Vehicle characterization (mass and geometry)

- Posttest data collection

* Test article permanent deformation

* Final position of test article

* Vehicle penetration depth

* Final resting point of vehicle

* Impact speed

* Damage estimate of vehicle

During analysis of the stated requirements, the team discovered additional requirements related to safety, reliability, efficiency, and repeatability. These additional requirements included the following.

- Stationary remote piloting location rather than piloting from chase vehicle

- Redundant wireless communication channels

- Heartbeat generator/listener to detect communication loss

- Test site isolated from people and buildings

- Test site isolated from radio frequency noise

- Remote kill switch

- Collect speed from global positioning system (GPS) receiver

- Automatic transmission (chose not to build remote shifter)

- Remotely pilot test vehicle (unmanned)

- Vehicle requirements

- Circular steering wheel with axis of rotation at center

- Good braking and acceleration

- Good steering

The requirement for stationary piloting location derives from previous experience remote piloting from a chase vehicle. In the chase vehicle experiments, proprioceptive inputs experienced by pilot in the chase vehicle were inconsistent with the dynamics of test vehicle making steering difficult and unreliable.

The incorporation of redundant wireless communication channels into the requirements allowed the system to mitigate effects of communication failure on a single channel. The heartbeat generator/listener would further mitigate complete communication loss by having the on-board 
microprocessor detect the communication loss and press the test vehicle brake, steer to neutral direction, and cut power to fuel pump.

The isolated test site would ensure safety for people and mitigate collateral damage to buildings in the event of failure of the remote piloting system. Radio frequency noise at the test site should be minimal to mitigate the risk of interference with wireless communication.

Two options available for measuring the velocity of the vehicle were from the On-Board Diagnostic II (OBD-II) or GPS. It was determined to measure speed with GPS because OBD-II was not available on all test vehicles. Furthermore, GPS provided a speed profile along the entire course, which was helpful for establishing braking distance, minimum course length required to achieve a specified speed, and more precisely control impact speed.

The implementation of the remote kill switch requirement ensured the vehicle had the benefit of quickly cutting power to the fuel pump and preventing the actuator from pressing the throttle pedal. These two actions are available in a single command from a kill switch and enforced in the microprocessor onboard the test vehicle.

The integration of the following components were required to fulfill the initial system requirement for RPVS.

- Gaming-type steering wheel and brake/throttle pedals

- Code written to interpret pilot commands from pilot controls

- Radio transceivers and high-gain antennae

- Microprocessor for interpreting driver commands and GPS data

- Driver actuator assembly from previous experiments

- Driver perspective dashboard camera

- GPS receiver

The system design of the RPVS fulfilled the initial requirements. The original design included a gaming-type force-feedback steering wheel with pedals that allowed the remote pilot to operate test vehicles up to the desired velocity.

Additional requirements were added to ensure quality and safety of the RPVS. These additional requirements are listed as follows: 
- Tasking language design (express throttle/braking/steering commands)

- Design heartbeat monitor

- Design for disengaging throttle and brake at impact

- Design of fail-safe logic

- Design of cruise control

- Modifications to driver actuator assembly

- Design of fail-safe braking system

- Design of mission monitor

- Developed protocol

The System Construction Stage consisted of building the RPVS to the requirements listed in the Requirements Analysis Stage. The construction and redesign of various versions of certain components were necessary during the life cycle of the RPVS. One example is the assembly of a shock and dust resistant case (18-in. x 11-in. x 16-in.) to house the electronic controls in the remote vehicle. The second example is the construction of antenna mounts and cables to connect the antennas to the radio frequency transmitters. Another example is the sizing and fitting of cables with connectors to link the transceivers and driver actuator assembly to the electronic control box.

The construction of a pilot station console was necessary for mounting the gaming steering and braking/throttle controls, which were fitted with a slip resistant footing. Appendix B lists a majority of the materials required to construct a RPVS.

The System Testing stage ensures the system of system performs as specified in the system requirements. This stage consisted of lab testing and field-testing with the RPVS. The conduct of the initial bench testing in the laboratory was necessary to confirm the receipt and execution of commands by the pilot (steering and braking/throttle). Laboratory testing of the system was required to ensure that the RPVS would successfully execute the kill switch command. Testing of vehicle dashboard camera was necessary to ensure the successful transmission of the wireless video signal to the pilot control monitor. Field-testing of the RPVS followed successful completion of bench testing. 
When performing field tests, the following concerns were addressed:

- A survey of the each test site was necessary to ensure clear communication at the longest extents of the test course, from the base station location to the barrier location. The longest range tested with negligible interference was $2000 \mathrm{ft}$.

- During remote piloting trials, the dash-cam video transmitter added too much latency to video for pilot $(>0.5 \mathrm{sec})$. This gave rise to a new requirement for faster (analog) video transmission.

- For each test, a calibration of the throttle/brake actuators was required for each vehicle as brakes/throttles engage differently on each vehicle.

- Pilot reported difficulty maintaining a given speed while controlling steering. The devised workaround allowed for the determination of the distance required to reach the target speed at full throttle. The vehicle's starting position was set at this distance from the barrier. This allowed the pilot to begin testing by pressing to full throttle throughout the test until impact. This approach proved not to be an optimal solution as some vehicles would sputter some distance along the test course before resuming the same acceleration as during trials. Furthermore, vehicles did not perform consistently on different days. This led to an additional requirement to implement cruise control. There was a discussion on whether to implement cruise control at the vehicle or at the base station. The final decision led to implementation of the cruise control at the base station; therefore, the vehicle would not have the capability to determine and set the throttle position autonomously. The implemented cruise control had controls similar to that on consumer automobiles through buttons on the pilot controls, meaning an onbutton to engage, pressing throttle or brake to disengage, or pressing off-button to disengage.

Each field testing session required lengthy setup and teardown. In addition, the sponsor indicated potential for additional tests in the following year. Further thought and discussion resulted in the decision to redesign the base station configuration. Components were concentrated into a compact, robust box allowing for semipermanent connections, and a common power supply could eliminate several small redundant power supplies. This simplified setup/teardown, reduced connection errors, and reduced wear on connectors and thus, maintenance. 
The Maintenance Stage consists of moving the RPVS from one vehicle to another after testing and continuously updating the system based on evaluation of its performance. This is also where the initial or current software changes occur. The continuous assessment of the system ensures that it does not become obsolete over its life cycle, while inspection of all components (e.g., transceivers, antennas, cables, circuits, dashboard camera, etc.) is necessary each time the system is used for crash testing.

The System Retirement Stage retires the systems and disposes of all unnecessary components. The RPVS has yet to reach this stage. 


\section{System of Systems Design}

This chapter provides the design details of the remotely piloted vehicle system (RPVS), which consists of five systems, i.e., (1) the vehicle system, (2) the base station system, (3) communication system, (4) test site and protocol for executing experiments, and (5) protocol for employment of system in experiments. This chapter provides a depiction and description of each subsystem and its components and discusses the underlying details.

The RPVS is a composite of people, products, protocol, test site, and processes that produce the capability of a remotely controlled vehicle, and allows for easy system installation into consumer vehicles with an automatic transmission. Figure 2 depicts the high-level design of the RPVS and its systems. Figure 3 shows a compact sedan with the RPVS installed. The RPVS consists of the procedures and people (denoted by the stick figures in Figure 2), the vehicle and control components (denoted by the red boxes in Figure 2), the base station control (denoted by the blue boxes in Figure 2), and the test site.

Figure 2. Remotely piloted vehicle system.

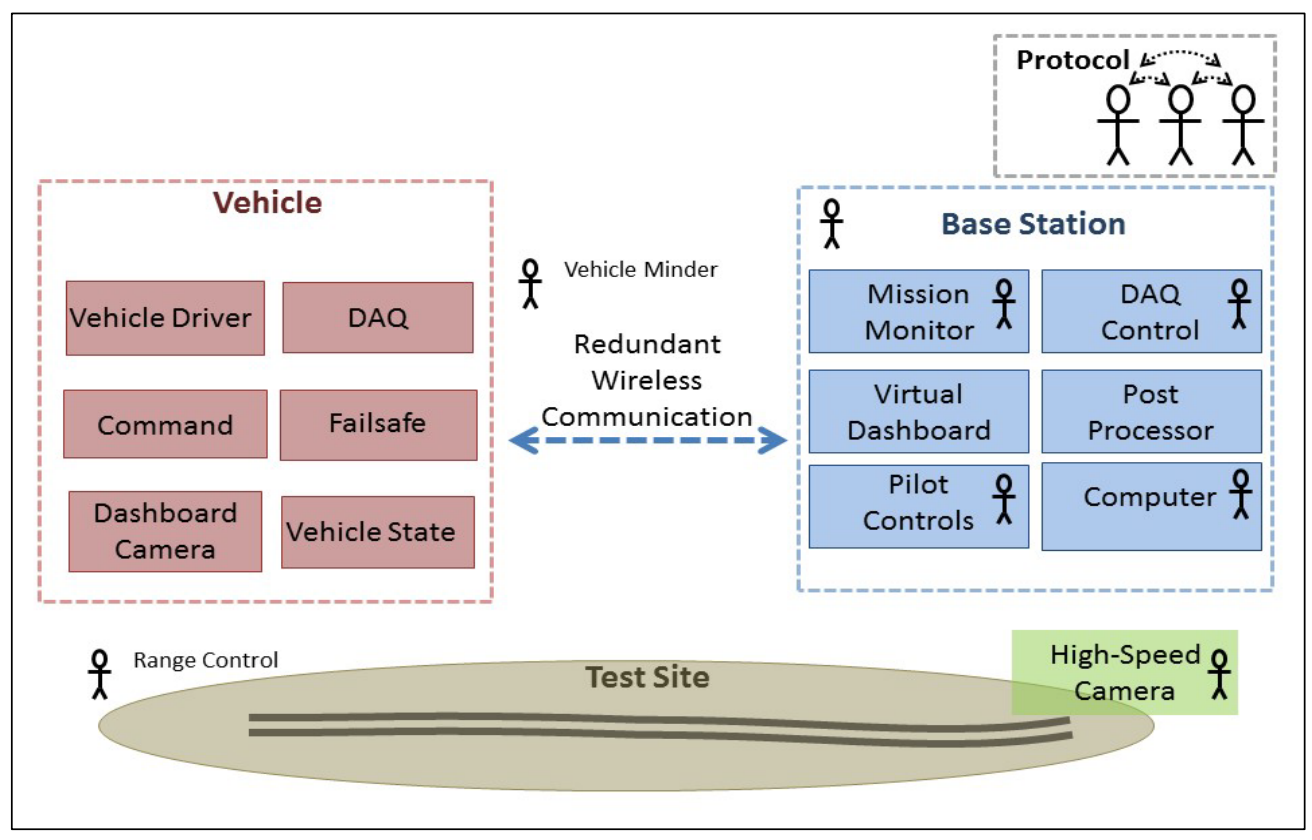


Figure 3. Example of vehicle with remotely piloted vehicle system installed.

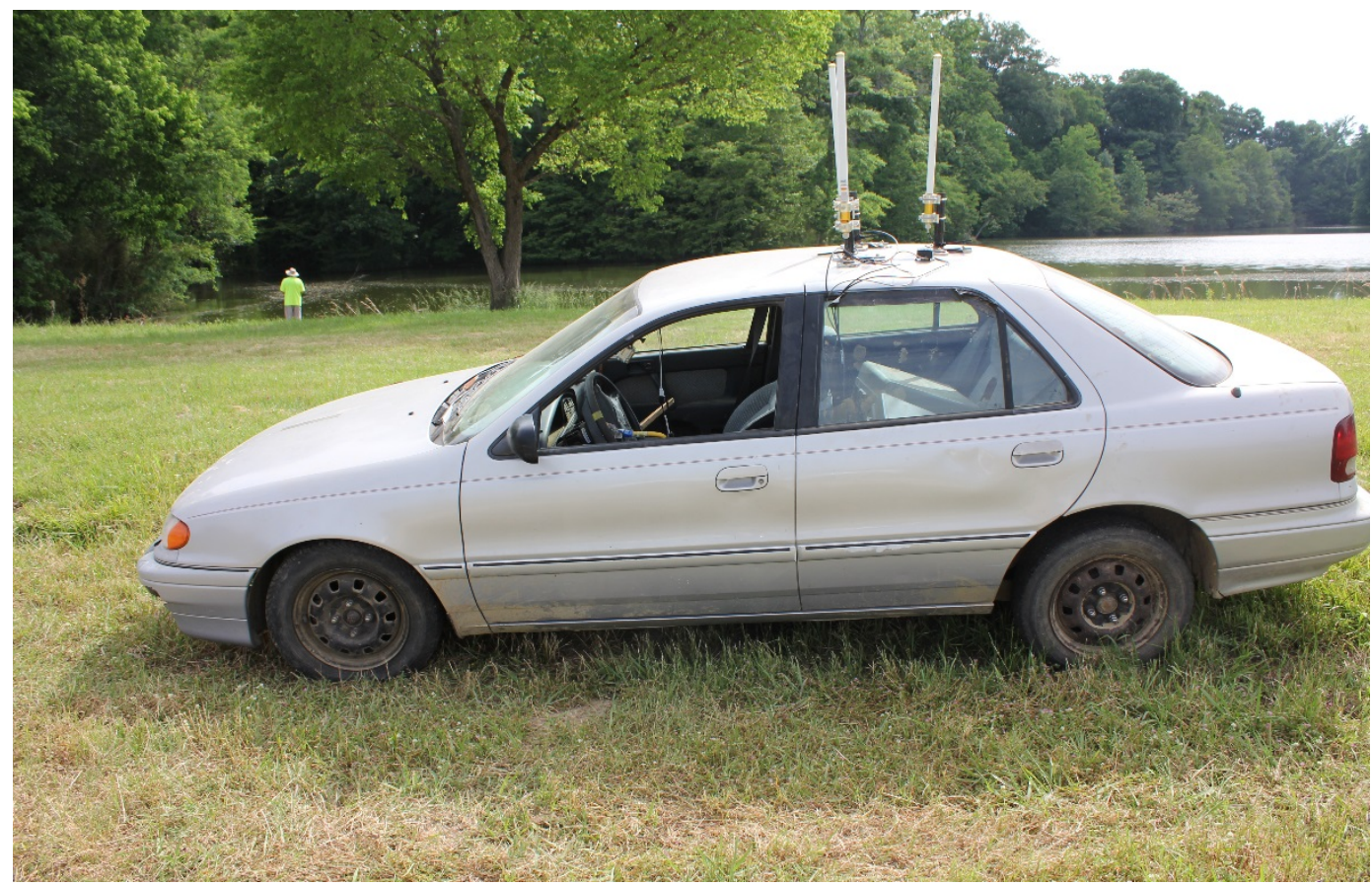

\subsection{Vehicle system}

The vehicle system consists of the following six components, i.e., (1) vehicle driver component, (2) the command component, (3) the dashboard video component, (4) the fail-safe component, (5) the vehicle state component, (6) the data acquisition (DAQ) component, and (7) the high-speed video component.

\subsubsection{Vehicle driver component}

The vehicle driver component is a kit installed in the test vehicle that enables the execution of the throttle and steering commands from the remote pilot. The vehicle driver component includes the following four features:

- Throttle/braking actuator

- Steering actuator

- Motor controller

- Actuator position sensors.

The throttle/braking actuator is the linkage between the vehicle driver and the test vehicle throttle and brake pedals. The purpose of this actuator is controlling the increase or decrease in speed of the test vehicle during 
operations. It is necessary to calibrate pedal controls for each vehicle. A procedure is available to determine and observe when the desired calibration level for full throttle and braking limits occur. These limits are then programmed at the base station to ensure that max throttle on the base station is max throttle on the vehicle. Without this calibration, it may be impossible to achieve max throttle in the vehicle or the mechanism may attempt to press throttle beyond the mechanical limit. This is also true for the brake limits.

Figure 4 shows the throttle/braking and steering actuators installed on a test vehicle. The steering actuator controls the steering angle, and the throttle/braking actuator presses the throttle/brake. Electric motors drive each of the actuators. A motor controller controls these electric motors to achieve a commanded steering and throttle/brake position of the test vehicle. The motor controller is able to get them to those positions by feedback from position sensors. The position sensors report current actuator positions so that error between current and commanded positions are available to the motor controller for converging this error to zero. A diagram of this is shown in Figure 5.

Figure 4. Braking, throttle, and steering actuator assembly.

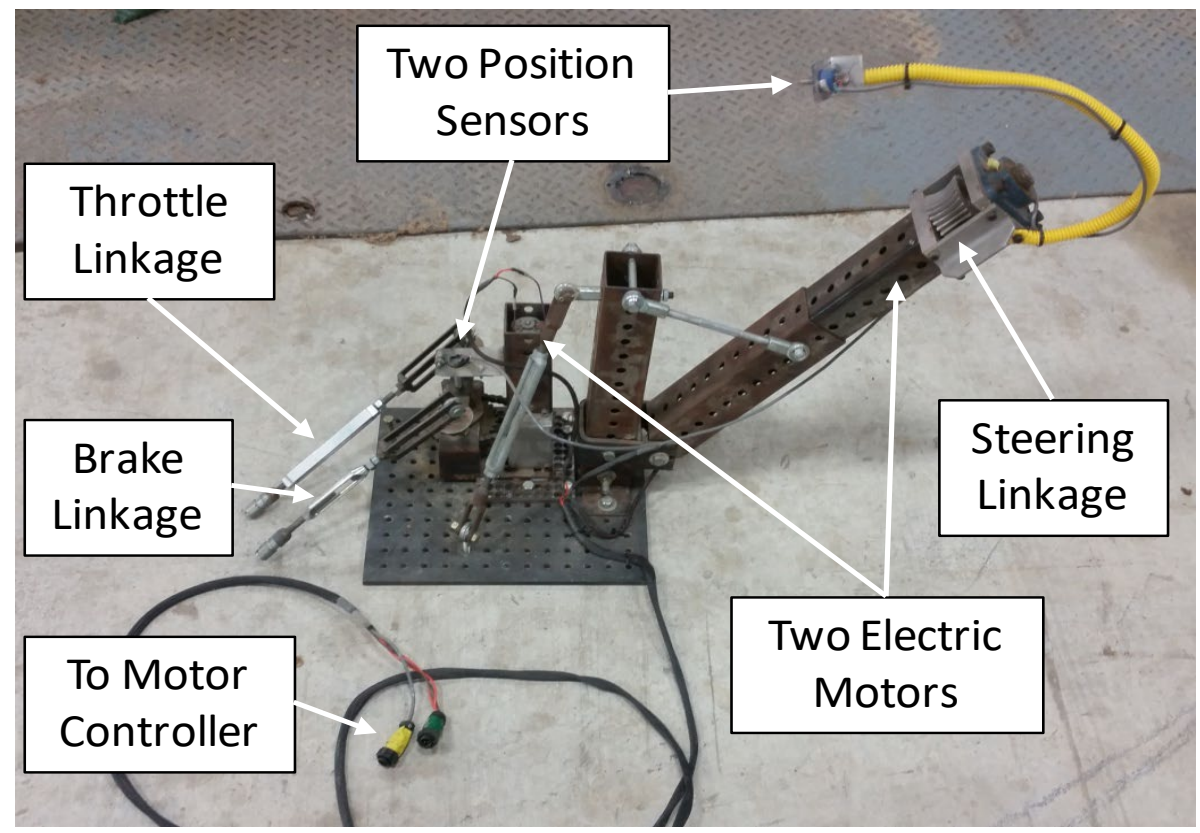


Figure 5. Diagram of actuator assembly connected to vehicle.

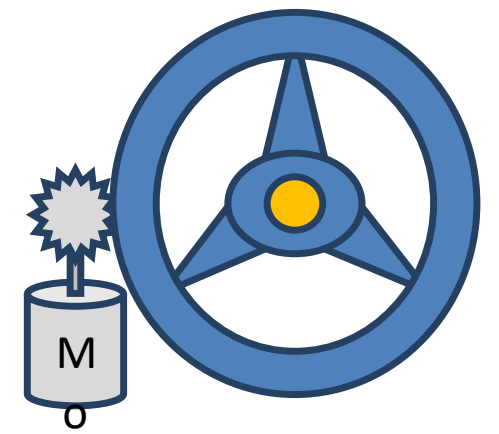

O Vehicle Controls
O Actuator Assembly
Position Sensors

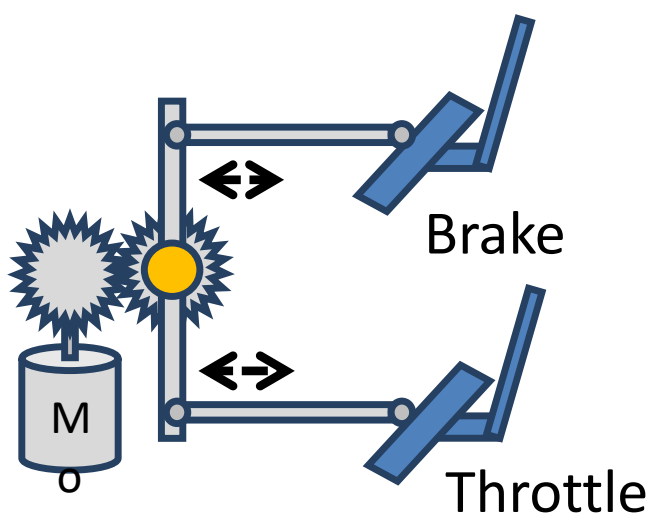

Throttle

\subsubsection{Command component}

The purpose of the command component is to process commands from the communication system and execute the most recent commands. The command component sends acknowledgements back through the communication system. The command component exists as software on a microcontroller in the vehicle.

\subsubsection{Dashboard video component}

The video component provides the remote pilot with the perspective from the dash camera. The video component includes two features:

- Dashboard camera

- Video transmitter.

The purpose of the dashboard camera is to provide the test pilot with a vehicle driving perspective similar to a driver sitting in the vehicle. Figure 6 shows the dashboard camera mounted in the center of the windshield of the test vehicle. The dashboard camera is a consumer camera that also has recording and audio capability used to document vehicle perspective remote piloting operations. A low-latency video transmitter sends the video signal from the dashboard camera to the base station. A monitor rated for outdoor viewing displays the video signal. 
Figure 6. Dashboard camera.

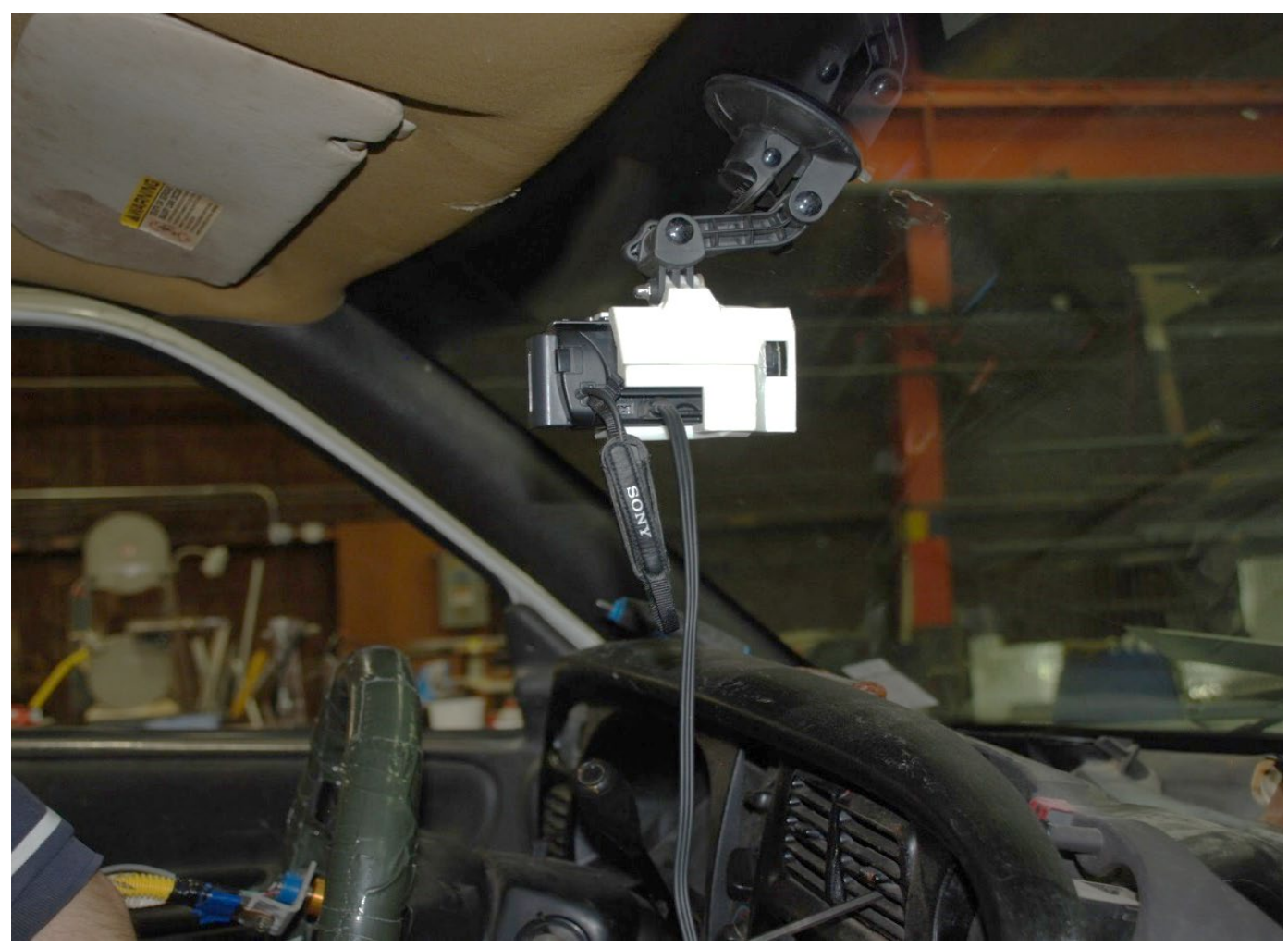

\subsubsection{Fail-safe component}

The fail-safe component ensures that the vehicle does not operate without positive control from the base station. It automatically shuts off the engine fuel pump and applies brakes when communication is lost between the vehicle and base station. The fail-safe component also shuts off fuel pump power immediately after detecting a loss of signal. Separately, the fail-safe component eliminates the capability of applying brakes upon vehicle impact with target barriers as required by test standards. The fail-safe component includes three features:

- Inertial switches

- Power to fuel pump

- Power to throttle/brake actuator

- Heartbeat listener

- Communication loss fail-safe

- Fuel pump relay and controller

- Throttle/brake actuator override system

- Control panel

- Status indicator

○ Engagement switch. 
The inertial switches provide an automatic fail-safe feature for the vehicle during the test. They enforce specific requirements for standard barrier performance testing (ASTM 2015).

The heartbeat listener monitors the status of the connection between the vehicle and base station.

The communication loss fail-safe consists of a relay placed in line with the power circuit to the fuel pump of the vehicle engine. In the event of communication loss, the state of relay changes to opened, which eliminates power to the vehicle fuel pump. Connected to the brake actuator is a separate relay that bypasses the motor controller and provides power to the brake actuator in the event of communication loss.

The control panel displays the state of the fail-safe system, activated or deactivated. Mounted on the control panel is the switch for engaging the fail-safe system.

\subsubsection{Vehicle state components}

The purpose of the vehicle state component is to sense and report speed and position states of the vehicle. The vehicle state sensor component includes six parts:

- GPS receiver

○ Speed

- Position

- Throttle/brake position sensor

- Steering positions sensor

- Motor controller

- Reporting system

- Data transmitter.

The purpose of the GPS is to provide vehicle position and speed during remote piloting operations.

The reporting system queries the motor controller for the throttle/brake and steering positions. It also collects and parses GPS receiver output. The reporting system reports these data via the data transmitter. 


\subsubsection{Data acquisition component}

The purpose of the data acquisition component (DAC) is to measure and record acceleration at different locations on the test vehicle, typically at the center of gravity (CG) and cargo areas of the test vehicle. The DAC component includes two features:

- Accelerometers

- Data logger.

The accelerometers provide data used to analyze the performance of the test vehicle during remote piloting operations. The data from the accelerometers are stored on a data logger.

\subsection{Base station system}

The base station system (BSS) and its components are the areas where the action of the system takes place. BSS consist of the following five systems, (1) mission display, (2) video monitor, (3) vehicle state display, (4) remote pilot controls, and (5) the DAC control. Figure 7 shows the base station system.

Figure 7. Base station control system.

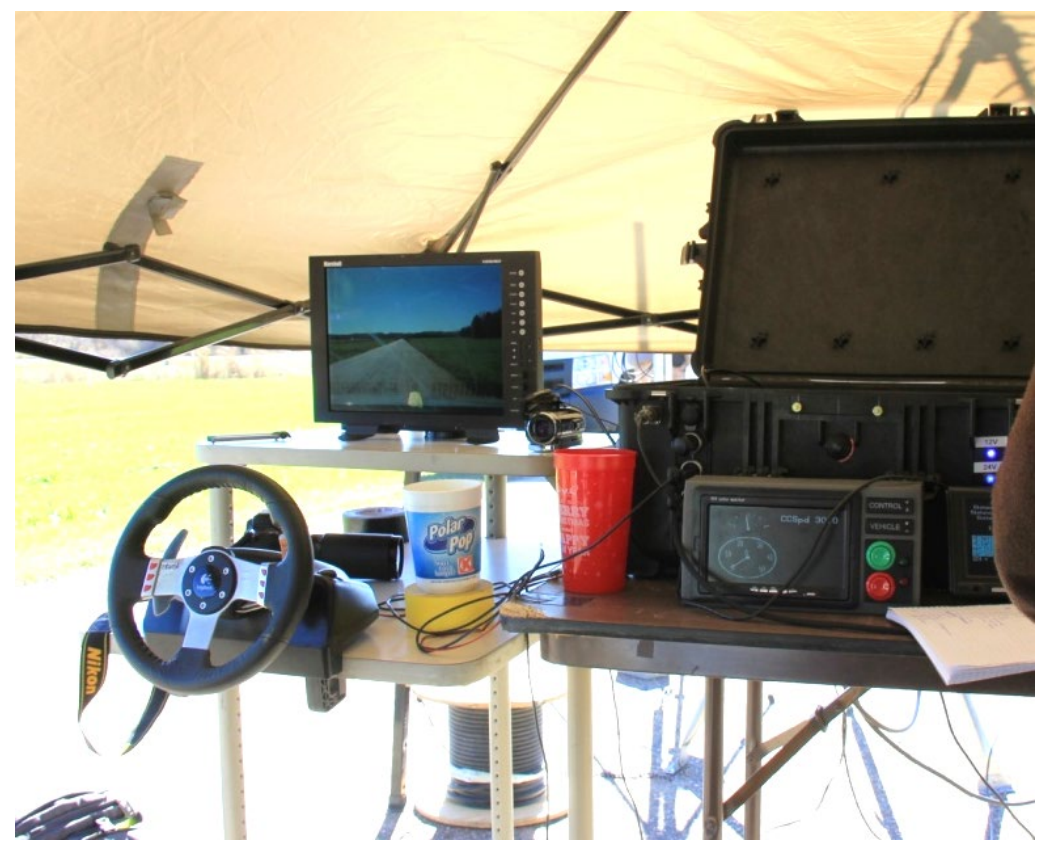




\subsubsection{Mission display}

The purpose of the mission display is to show real-time diagnostics, the state of the communication channels, and speed and distance from the GPS.

The mission display component includes three features: (1) communication, (2) GPS, and (3) diagnostics.

Figure 8 shows the mission monitor that displays the distance traveled, the distance to impact in feet, the distance to the position of no return (PONR) in feet, the speed in miles per hour, and the elapsed time in seconds.

Figure 8. Mission display.

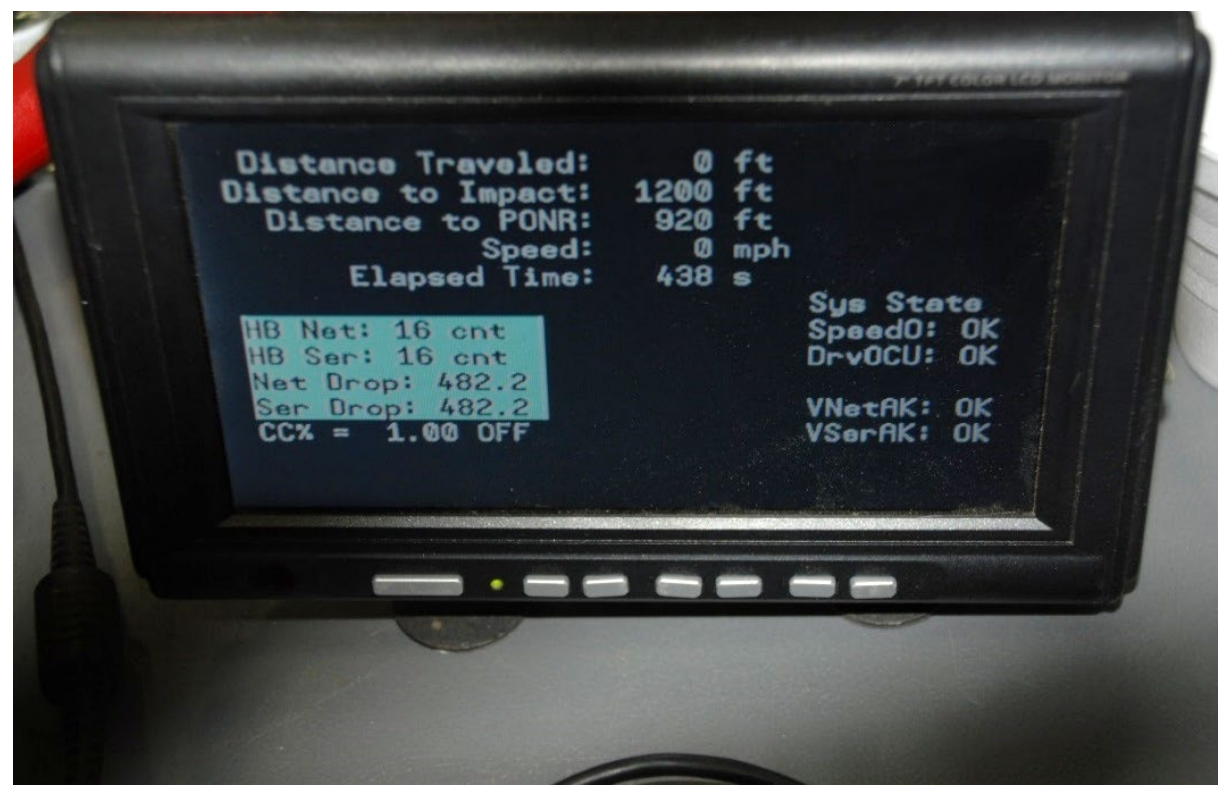

\subsubsection{Dash cam display}

The dash cam display is a monitor displaying video from the dashboard camera perspective from the inside of vehicle.

Figure 9 shows the dash cam display, which is responsible for providing the test pilot with a dash cam view. 
Figure 9. Dash cam video display.

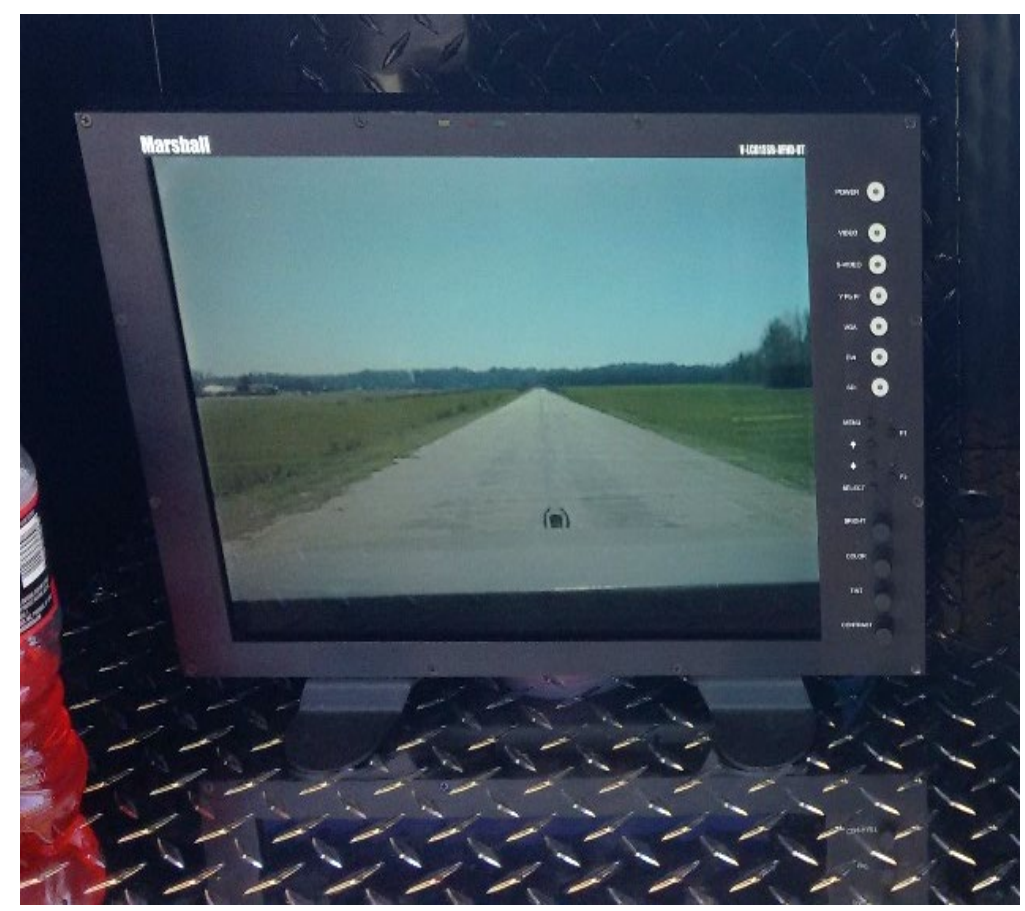

\subsubsection{Vehicle state display}

The vehicle state component includes three features: (1) cruise control, (2) speed, and (3) throttle position. Figure 10 shows the vehicle state display. The role of the vehicle state display shows the cruise control target speed. The vehicle state display provides the mission controller with verification that the programmed vehicle operate at the desired speed.

The mission monitor observes the speed gauge on the display during the test to determine if the vehicle is on track to reach target speed upon impact.

The purpose of the throttle position gauge is to verify the vehicle is responding to throttle and brake commands during pretrial tests. Another purpose of the gauge is to verify that the throttle/brake actuator behaves as expected. 
Figure 10 . Vehicle state display.

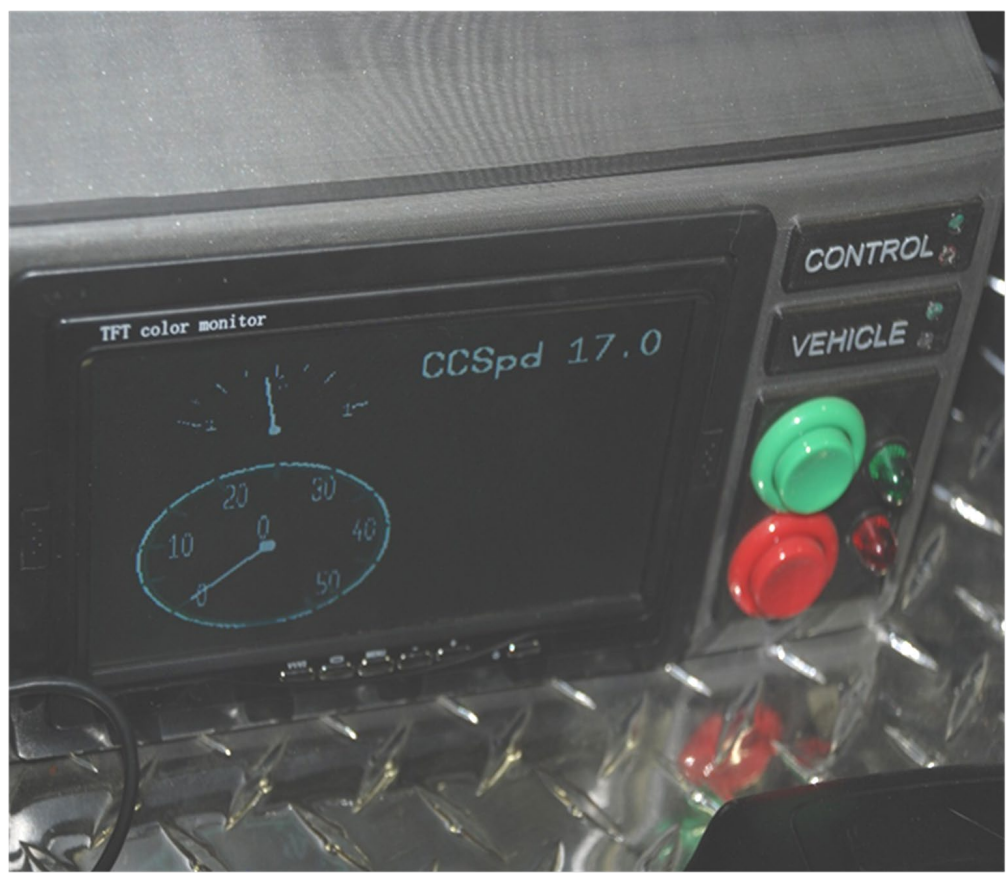

\subsubsection{Remote pilot controls}

Figure 11 shows an example of the remote pilot base station. The remote pilot controls consist of a steering wheel, brake and throttle pedals, kill switch button, and cruise control on/off buttons (any pedal movement also turns off cruise control). The remote pilot control is a remote human interface device that provides human control for the remote vehicle.

Figure 11. Remote pilot controls.

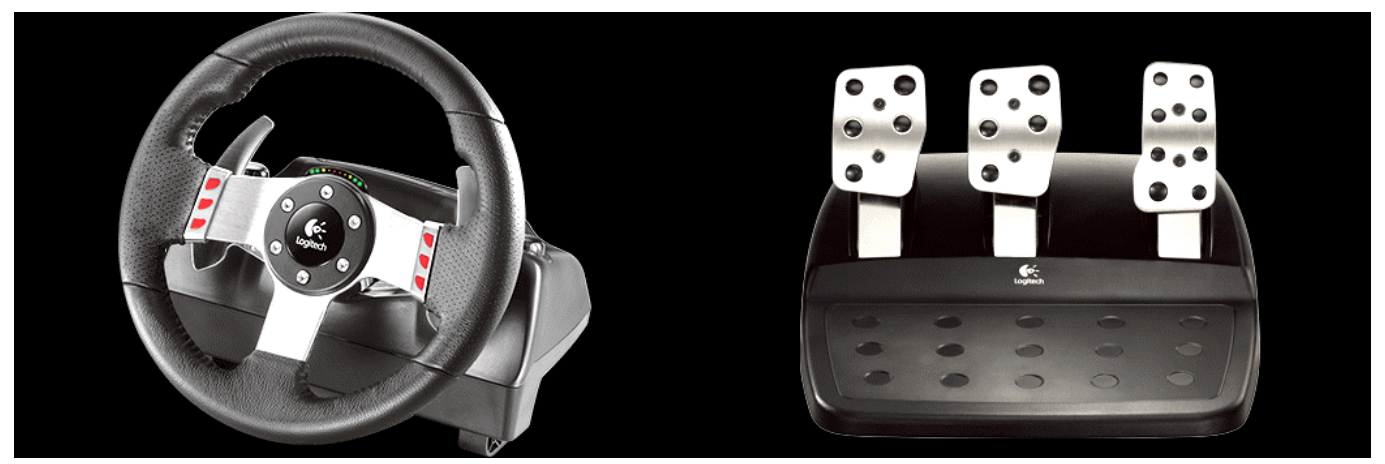




\subsubsection{DAQ control}

The purpose of the data acquisition component (DAQ) is to measure and record acceleration at different locations on the test vehicle, typically at the center of gravity (CG) and cargo areas of the vehicle. The DAQ component includes two features, accelerometers and data logger.

The data provided by the accelerometers serve as a tool to analyze the performance of the test vehicle during remote piloting operations. The data from the accelerometers are stored on a data logger.

\subsection{Test site}

The test sites for this research vary between tests and are mission related. The site must be relatively flat and smooth. The length of the site varies from approximately $800 \mathrm{ft}$ to $2,000 \mathrm{ft}$. The work area must be secure, covered, and have electricity or a power source such as a generator.

Figure 12 shows an example of a test site. Jersey barriers, forests, or open areas must limit the free-roll zone. ASTM standards require high-speed video components for documenting performance data during high-speed impacts. Figure 13 shows the high-speed video camera setup in the field.

To date, test sites have been in Vicksburg, MS and Fort Lenard Wood, MO.

Figure 12. Test site includes base station, vehicle, people, and communication components.

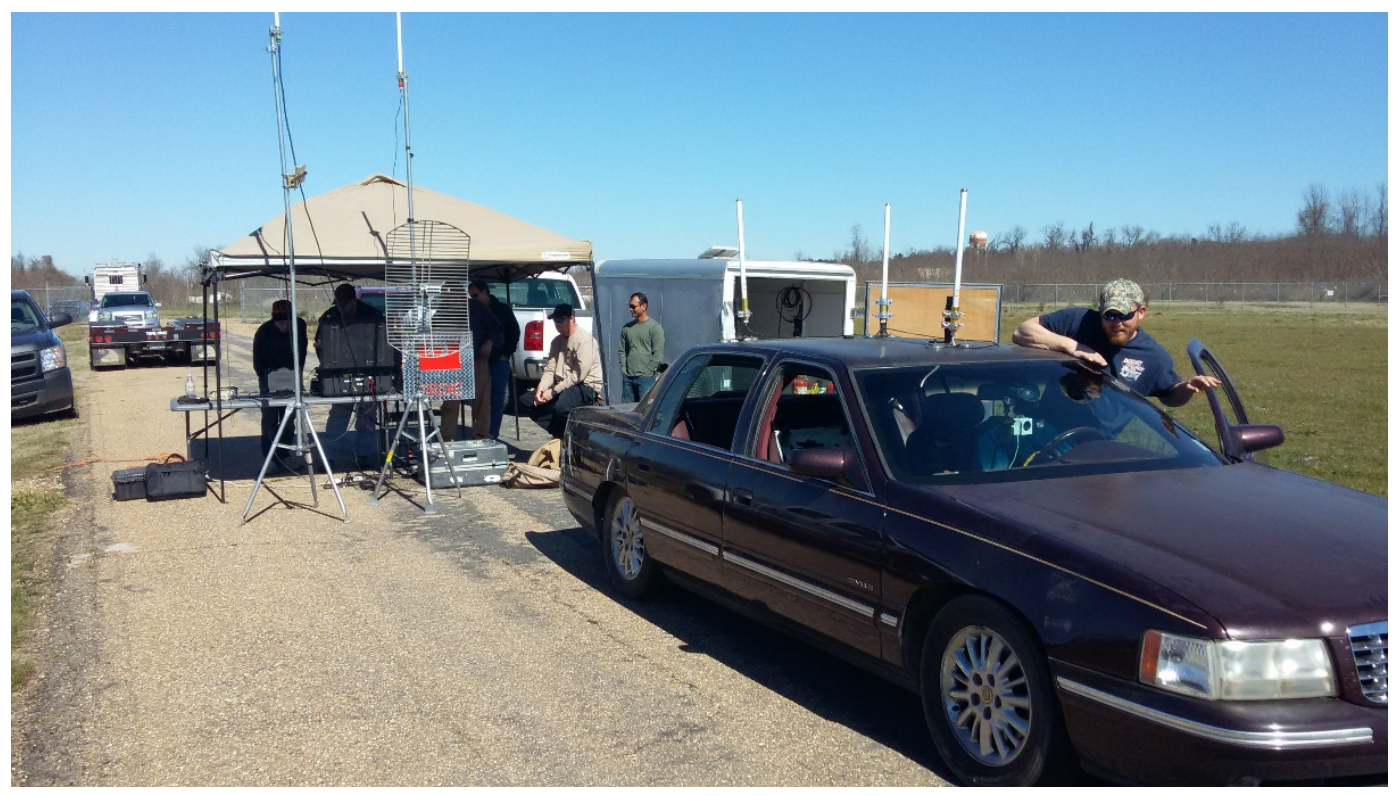


Figure 13. High-speed video.

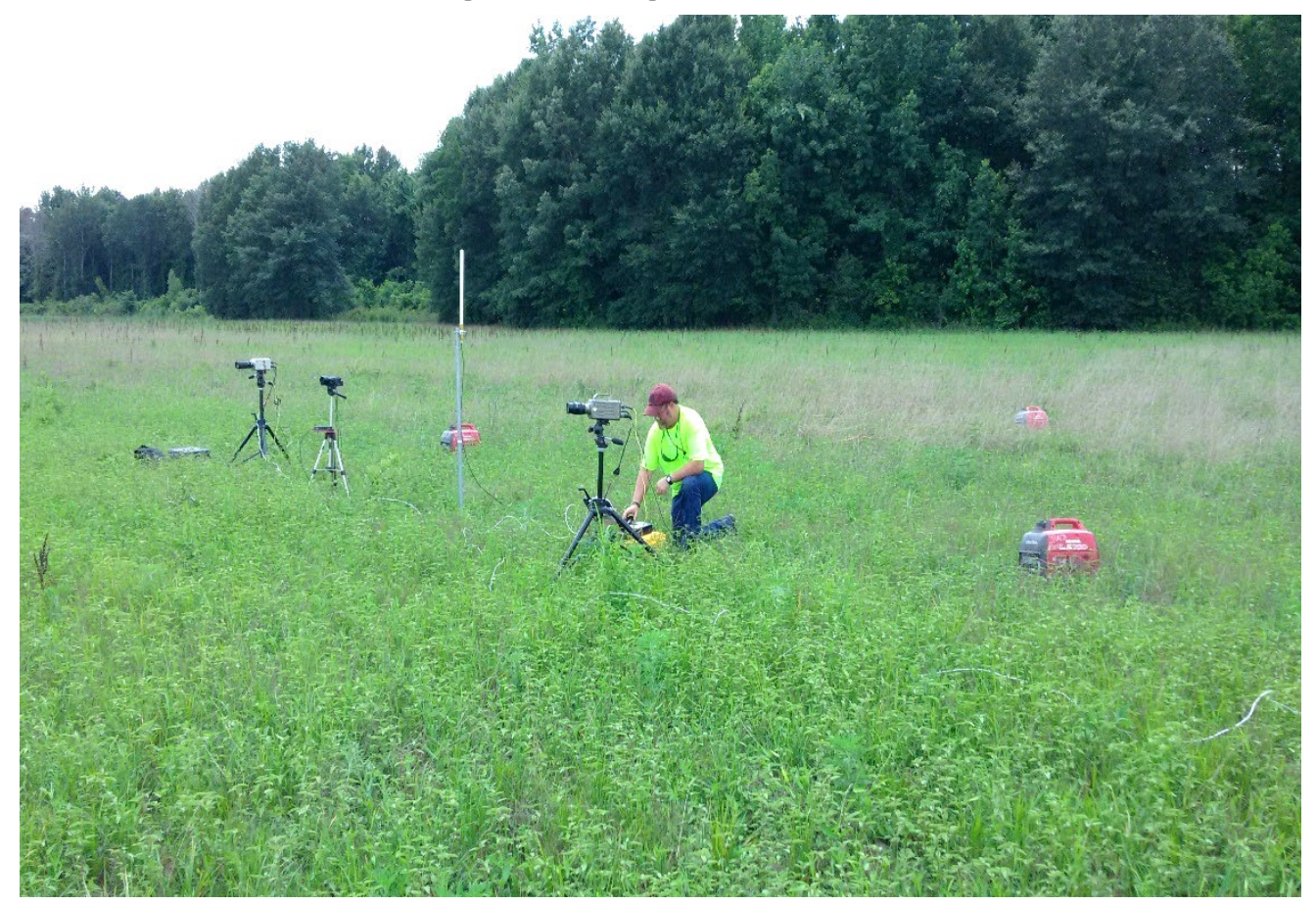

\subsection{Protocol}

Each test requires a series of personnel to act in varying roles during the test execution. The required roles are (1) vehicle minder (VM), (2) mission monitor (MM), (3) test pilot (TP), (4) range control (RC), (5) mission control (MC), and (6) the high-speed video (HV) controller. Figure 14 shows the personnel and their roles.

The VM is responsible for readying the vehicle for test commencement.

The MM is responsible for monitoring the mission parameters (speed, distance to impact, etc.) during test execution.

The TP is responsible for remotely operating the vehicle during test execution.

The $\mathrm{RC}$ is responsible for securing the test site and ensuring the safety of the vehicle for approach after impact.

The $\mathrm{MC}$ is responsible for ensuring the overall test execution and initiating and managing test protocol. 
The HV controller is responsible for setting up the high-speed cameras and starting/stopping them at appropriate time during test execution.

The safety protocol ensures that testing does not commence with personnel down range and ensures that all systems are ready for go. The safety protocol also ensures that the test vehicle is safe for approach after impact.

For each test, there is protocol, when followed that ensures safety, an effective test execution, and equipment operation.

Figure 14 shows the test protocol sequence along with the role players.

Figure 14. Test protocol sequence.

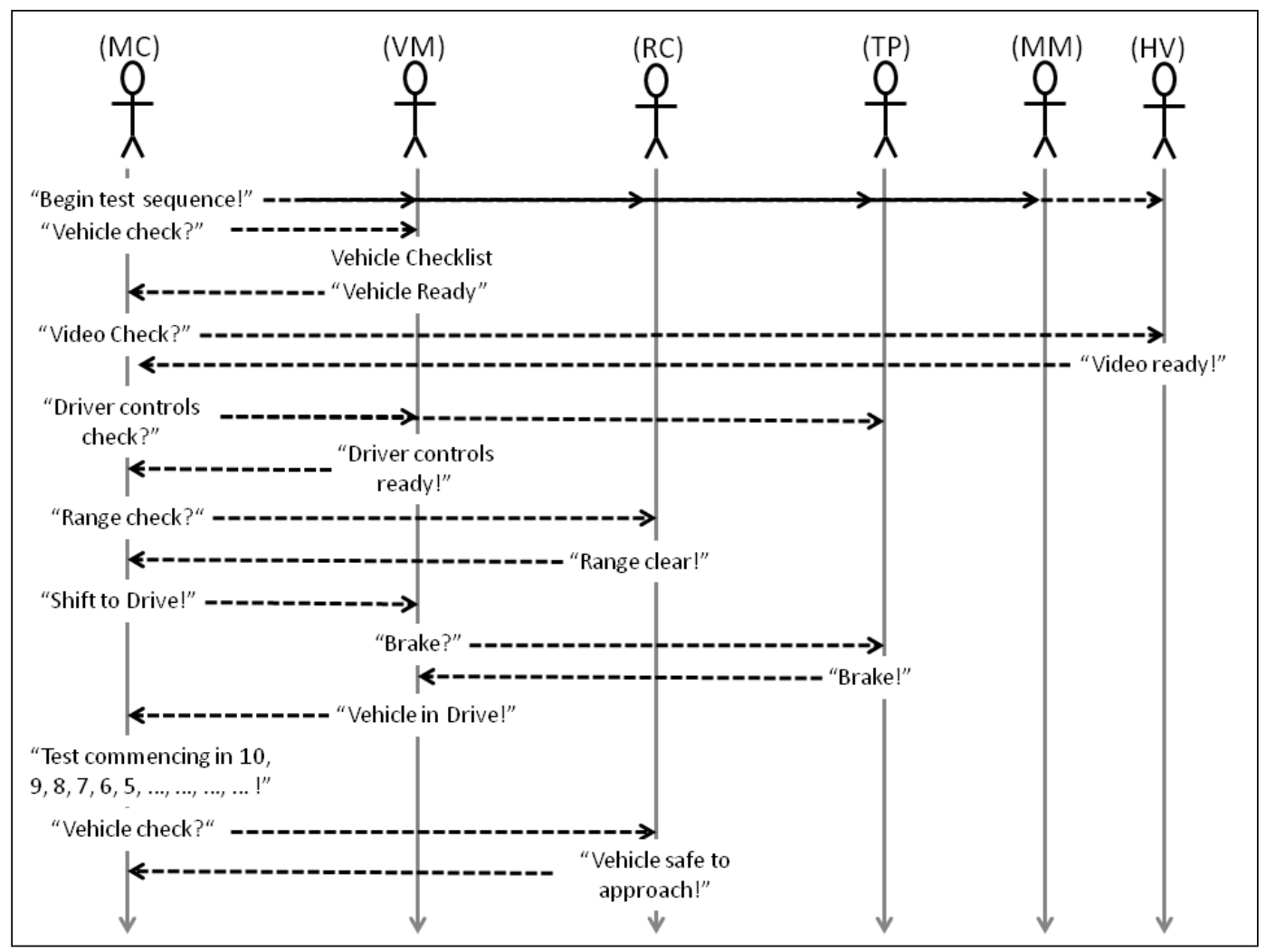




\section{System Perspectives}

\subsection{Driver perspective system}

The driver perspective system provides the test pilot with a "first person" view through the windshield to operate the test vehicle through the video display. This system also provides a virtual dashboard to the test pilot. Displayed in the virtual dashboard are gauges similar to what a driver would see in an actual vehicle. Figure 15 displays components of the driver perspective system and their disposition at the vehicle and base station as well as communication channels.

Figure 15, Driver perspective of system.

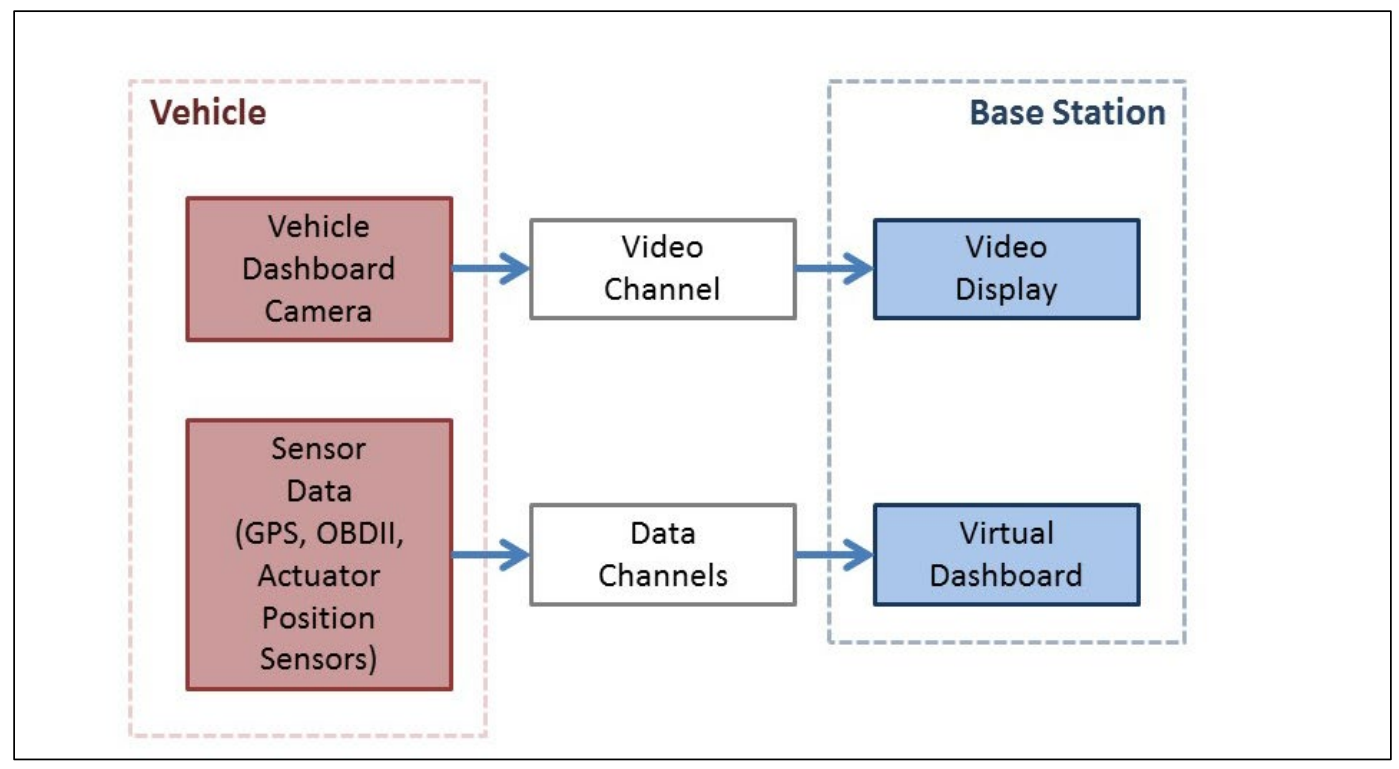

\subsection{Instrumentation system}

In order to ensure the test objective milestone, the instrumentation system collects and logs data. This includes monitoring the vehicle up to target velocity and after impact. The data allow for the assessment of the overall performance of the RPVS during experiments.

Figure 16 shows the components of the instrumentation system. The diagram is color coded where red represents components on the vehicle, blue represents components at the base station, orange are test site components, and white represents the communication components. The arrows represent the direction of dependency between components. 
Figure 16. Instrument perspective.

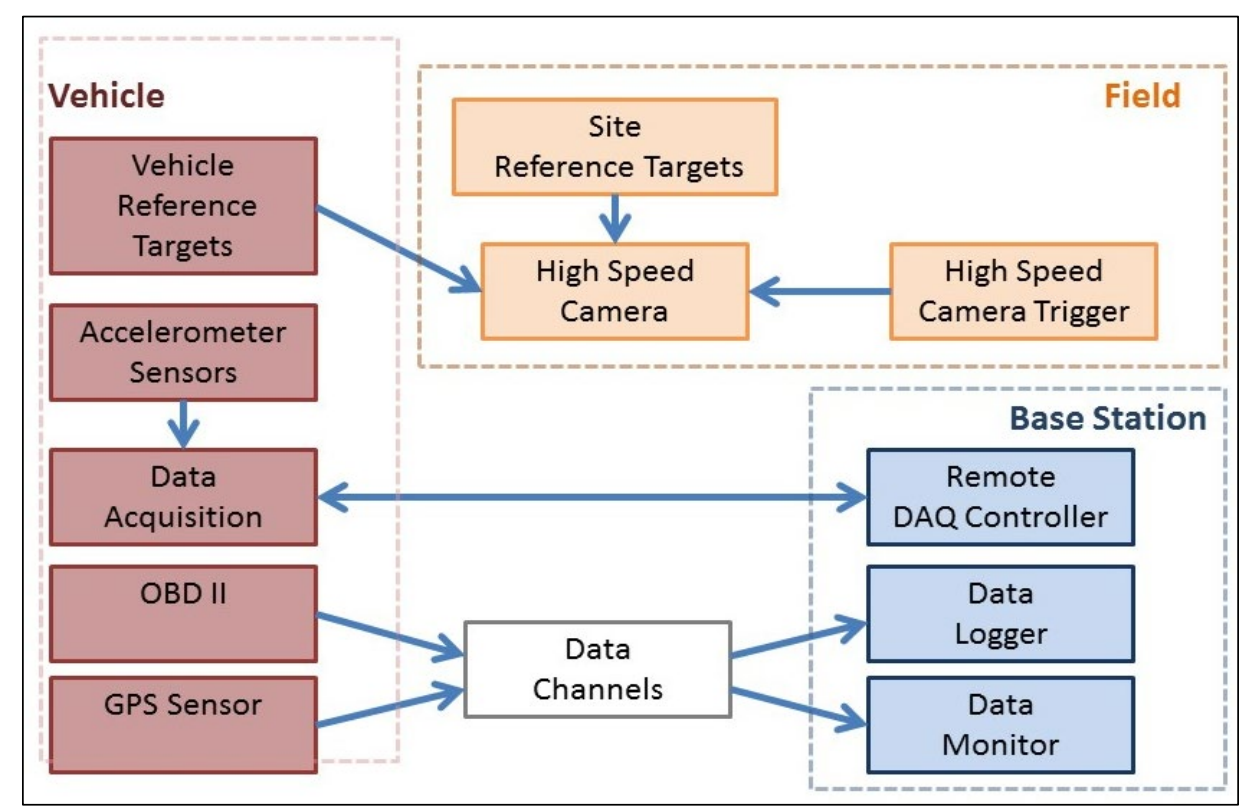

Site reference targets are stakes placed at predetermined intervals to allow for analysis of the high-speed video. The site reference targets (like a checkered board) are placed near the tested barrier to determine vehicle speed at impact.

Vehicle reference targets are adhered to the vehicle for high-speed video analysis. The high-speed camera trigger starts the recording of the highspeed camera. The high-speed video controller or a sensor triggers the high-speed camera.

\subsection{Piloting system}

The piloting system allows the test pilot the ability to operate the test vehicle remotely. The system relays commands from the test pilot and executes the commands in the test vehicle. The commands are decoded and executed via the motor controller that allows the system to engage the actuator assembly, which controls the brake, throttle, and steering of the test vehicle. Figure 17 shows the components of the piloting systems. The diagram is color coded where red represents components on the vehicle, blue represents components at the base station, and white represents the communication components. The arrows represent the direction of dependency between components. 
Figure 17. Piloting system perspective.

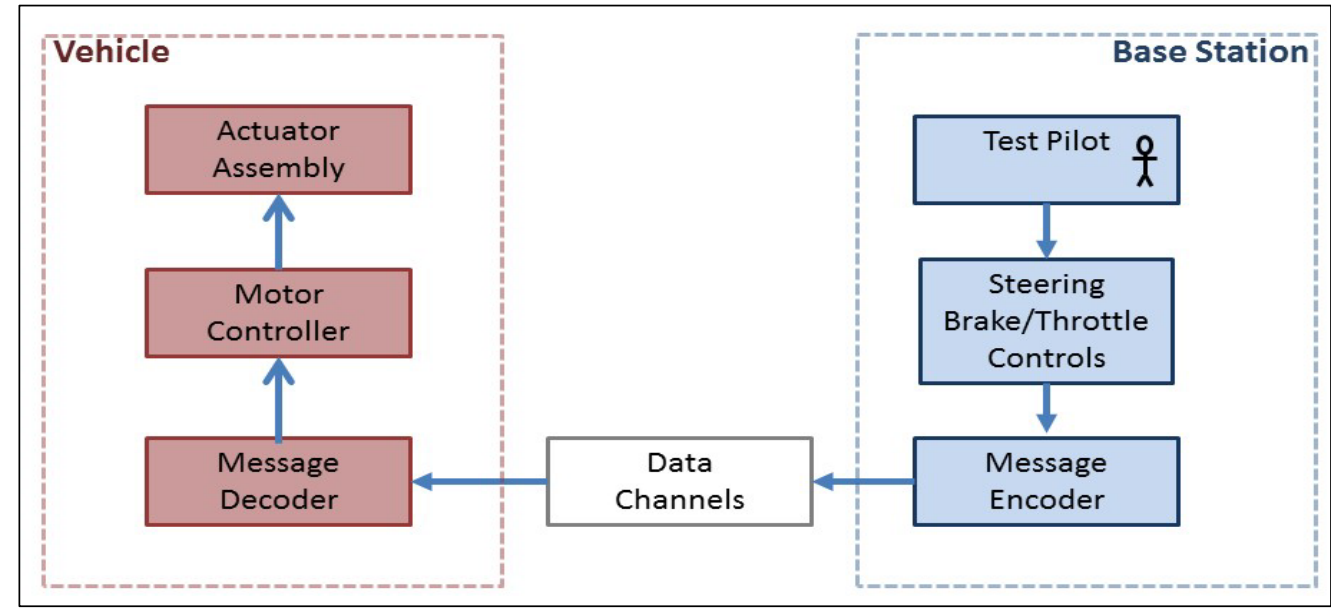

\subsection{Safety system}

The safety system is a combination of measures to minimize the risk of failure. These measures include electronics, watchdog monitors, and protocols for test personnel. The safety system ensures that the vehicle does not operate without positive control from the base station. The safety system also allows the test pilot to remotely shut off the engine. The safety protocol ensures that testing does not commence when personnel are down range and ensures that all systems are ready for go. The safety protocol also ensures that the test vehicle is safe for approach after impact. Figure 18 shows the safety system perspective. 
Figure 18. Safety system perspective.

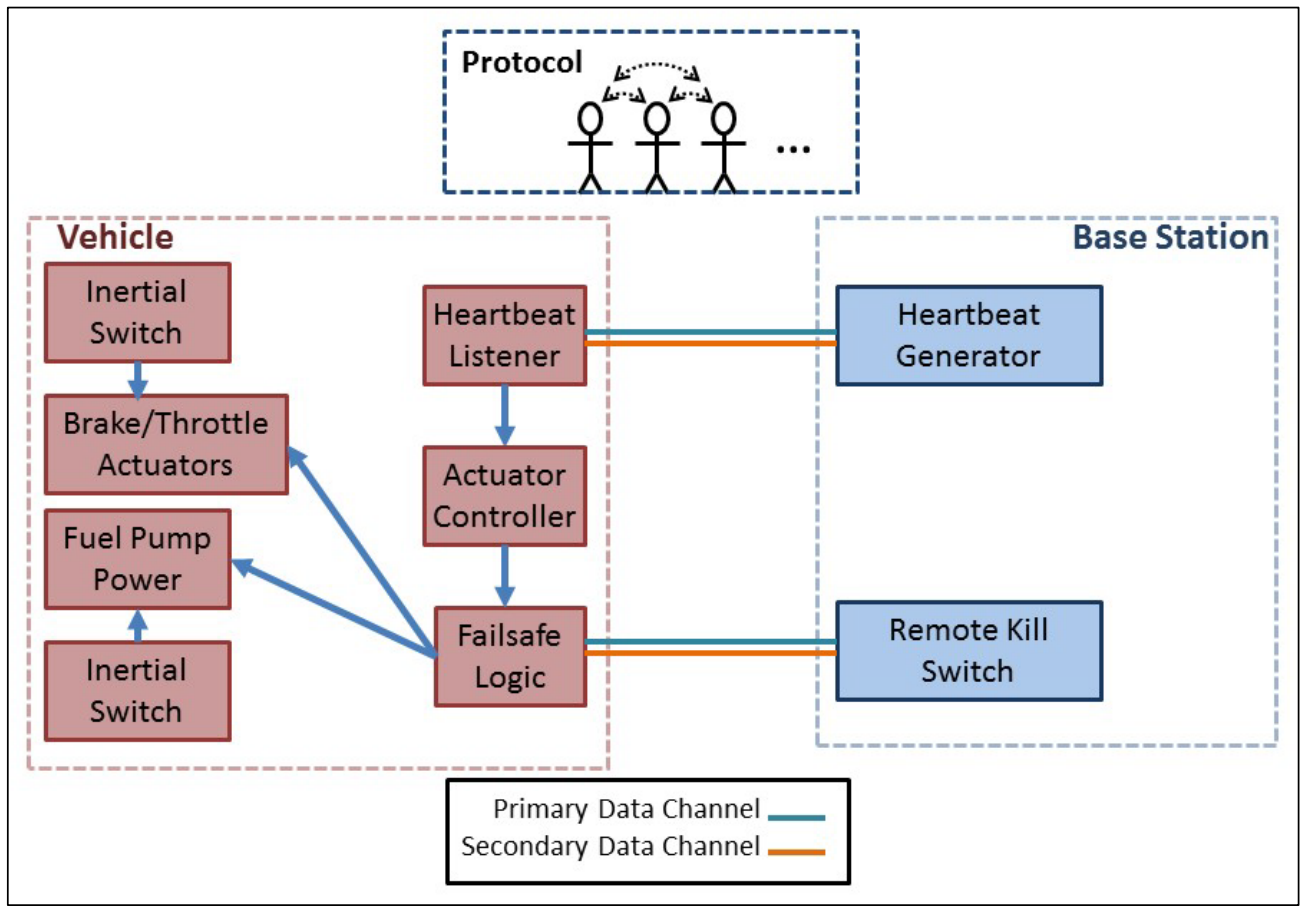

\subsection{Communication}

The purpose of the communication system is to provide reliable wireless communication between vehicle and base station. Figure 19 shows the communication perspective of the system. The system includes radio transceivers, a messaging protocol, a tasking language, and a manager to execute communications.

The ruggedized wireless transceivers perform the telemetry. One set of transceivers is for $\mathrm{Wi}-\mathrm{Fi}$ at $2.4 \mathrm{GHz}$, which is the primary communication channel due to a low latency requirement. The configuration of the Wi-Fi transceiver transport is the user datagram protocol (UDP) instead of transmission control protocol (TCP) to prevent the persistence of obsolete messages. The second set of transceivers is for serial communication at $920 \mathrm{MHz}$. The serial transceivers are not as fast as Wi-Fi but have a longer range and drop out less frequently. The radios transmit through high-gain antennas. The base station antennas are directional, and the vehicle antennas are omnidirectional. 
Figure 19. Communication perspective of system.

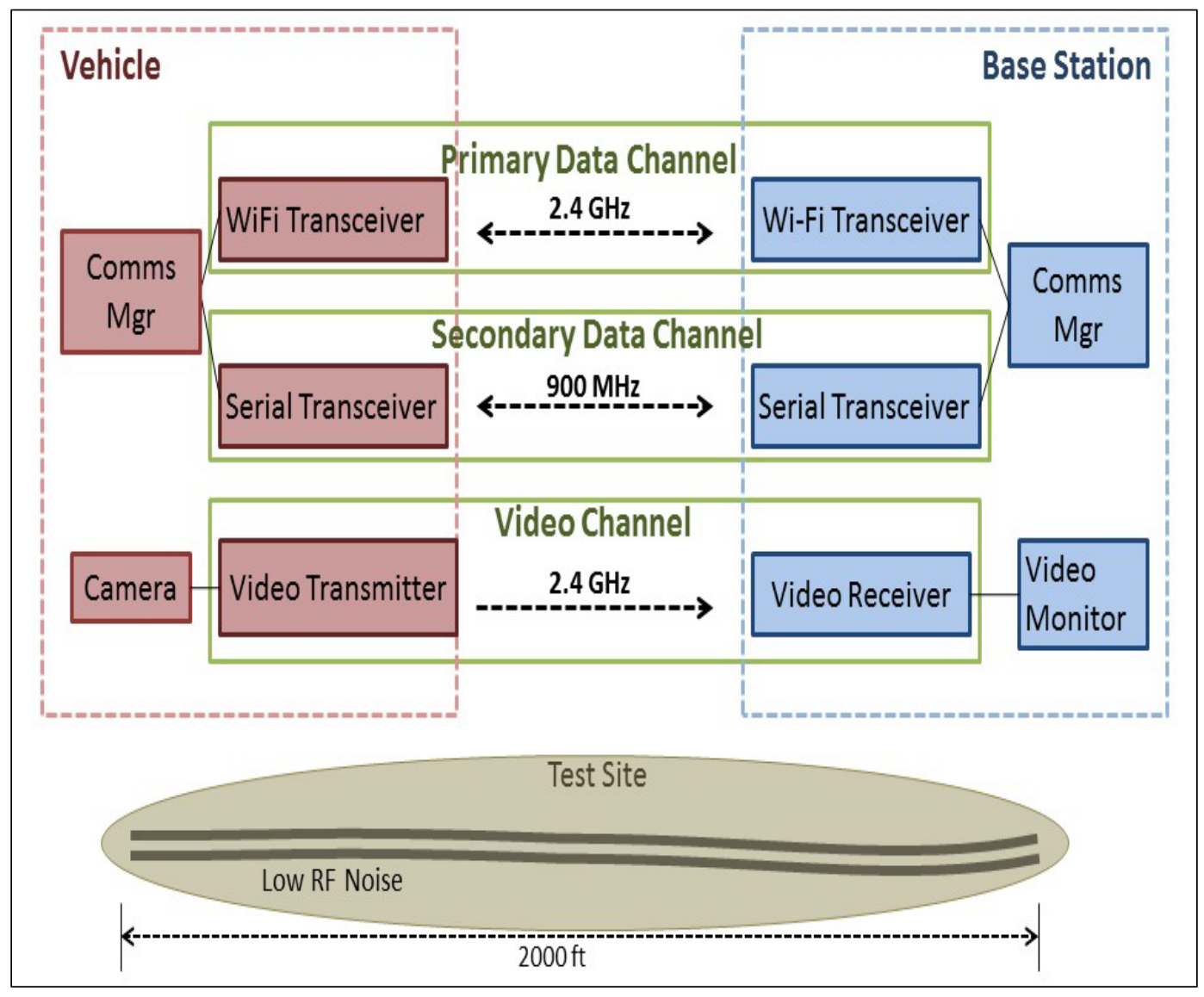

There is a protocol for encapsulating messages. The syntax includes the \# symbol to begin a message. The message content is next, followed by a four-character hexadecimal sequence number that ensures the execution of the messages in sequence. The message is concluded by a ! symbol if the message is a request and a $\$$ symbol if it is a response. An example message is shown as follows.

$$
\# D, 7 b, 82,0 F 20 \text { ! }
$$

There is also a tasking language for communicating messages between components. There are 10 different types of request/response commands enumerated in the following list.

- Steering $=\mathrm{A}$

- Throttle/brake = B

- Steering/throttle/brake $=\mathrm{D}$

- Heartbeat $=\mathrm{C}$

- Control status query $=\mathrm{P}$
- $\quad$ Fuel set state $=F$

- GPS update $=\mathrm{G}$

- OBD update $=\mathrm{O}$

- $\quad$ Kill command $=\mathrm{K}$

- Initialization = I 
The communications manager monitors both channels (Wi-Fi and Serial) and sends/receives command messages that are decoded from the messages. When receiving messages, the communication manager arbitrates for the most recent message from either the Wi-Fi or Serial channel. 


\section{Application of RPVS}

The RPVS is applicable for any remote driving experiment. However, this chapter describes employment of RPVS for barrier testing and captures the actual actions to prepare for test day activities for the RPVS. Employment of the RPVS requires a coordination of multiple activities, personnel, procedures, and equipment.

\subsection{Experiment site survey}

The purpose of the site survey is to determine suitability for conducting remote driving operations. The requirements are (1) smooth level test track, (2) adequate area for remote driving, (3) features to contain test vehicle, (4) covered workshop with power, and (5) low noise within transmitter bands.

\subsection{Experiment site setup}

Appendix D provides six checklists for each phase of an experiment. The checklists are T-minus 24 hour, packing, vehicle setup, test range, vehicle barrier test, and take down. Each checklist includes the items needed and the steps required for that phase.

\subsection{Rehearsal}

To ensure that all systems of the RPVS are working, a pretest of the remote driving operations is conducted several days in advance of the actual experiment. If any problems occur, the implementation of an immediate solution must occur before test execution. Refer to Appendix $\mathrm{C}$ for more details on rehearsal experiments.

\subsection{Execution}

The actual test takes less than 2 min.

\subsection{Data archival}

The data archive records data from each system are as follows.
1. GPS
4. UDP
2. Braking/throttle
5. Serial
3. Steering
6. Acceleration 


\subsection{Reporting}

Generated from the data is a series of charts for each reporting system. Shown in Chapter 6 are samples of the data from various tests.

\subsection{Test site breakdown}

When test is completed, the vehicle system, base station, and all test site components are broken down and transported back to a local facility for storage and maintenance if needed. 


\section{Performance Metrics}

\subsection{Speed and distance, steering, throttle}

The design of the RPVS required the vehicle to achieve a target impact speed and to stop within a controlled distance. The steering of the RPVS must be responsive and accurate enough to operate the vehicle safely along the test course. The throttle and brake also needed to be responsive and accurate enough so that the vehicle promptly executes commands from the test pilot.

Figure 20 shows four charts including speed and distance curve, communication and kill event, steering curve, and throttle/brake curve.

The speed and distance curves represent data recorded from the GPS on the vehicle. The speed curve represents the test vehicle velocity during remote driving operations, and the distance represents the distance the vehicle traveled since the last startup time of the remote piloting software. The black horizontal line on this chart represents the minimum speed the vehicle must achieve for test events. The vertical black line intersects the speed and distance curves at the targeted minimum speed for testing.

The event chart displays the communication latency of the UDP (Wi-Fi) and serial channels. Small red and green dots depict communication; their channels are indicated on the vertical axis. The further away the dots are from each horizontal axis indicates a higher latency. A gap in the dots indicates a failure in that communication channel. The kill signal, red dot, displayed on this chart indicates engagement of the kill switch by the remote pilot.

The steering curve represents two different types of steering data. The data from the steering commands sent from the remote control steering wheel are plotted in the third chart in Figure 20. Figure 20 depicts data recorded and plotted from the position sensor located on the vehicle steering wheel along with the remote control steering commands.

The throttle/brake curves depicted in Figure 20 illustrate which position the test vehicle throttle/brake positions are relative to the remote control throttle/brake positions. Also depicted in Figure 20 is the cruise control event, represented by the black vertical line. 
Figure 20. Remotely piloted vehicle system data (11/12/2015 test).

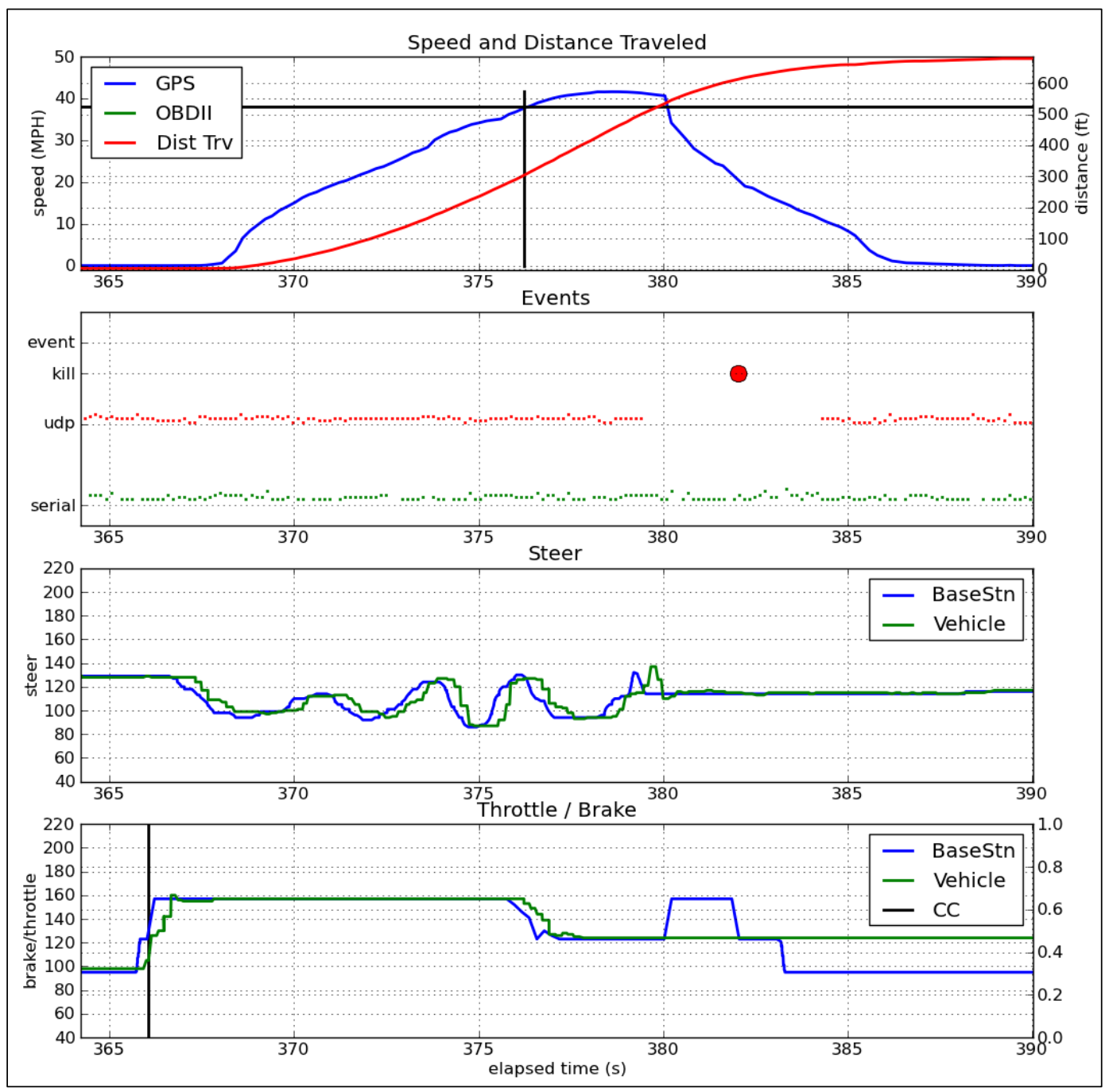

At time 365 on the throttle/brake chart, the vehicle is sitting at the start point with the brake applied. At $\mathrm{t}=366$, the brake is released, and cruise control is engaged. The throttle is applied under cruise control from the base station. At $t=367$, the actuator in the vehicle applies throttle, and the vehicle accelerates. During the test, the remote pilot makes steering adjustments. At $t=376$, the minimum impact velocity is achieved and the cruise control begins letting off the throttle. At $t=380$, the vehicle impact occurs as indicated by the drop in speed. Note that at this time, the udp communication is lost likely due to impact as depicted by the break in the red dots on the events chart. The serial channel remained unaffected by the impact. The cruise control attempts to compensate by sending full throttle commands. However, in the event of the inertial switches tripping by impact of the vehicle with the barrier, the actuator does not apply throttle and remains at a neutral position as designed. At $\mathrm{t}=382$, the 
remote pilot sends the kill command, depicted by big red circle, which disengages the cruise control and removes power from the fuel pump in case the inertial switch was not tripped by impact.

Figure 21. Trial test 7/28/2014.

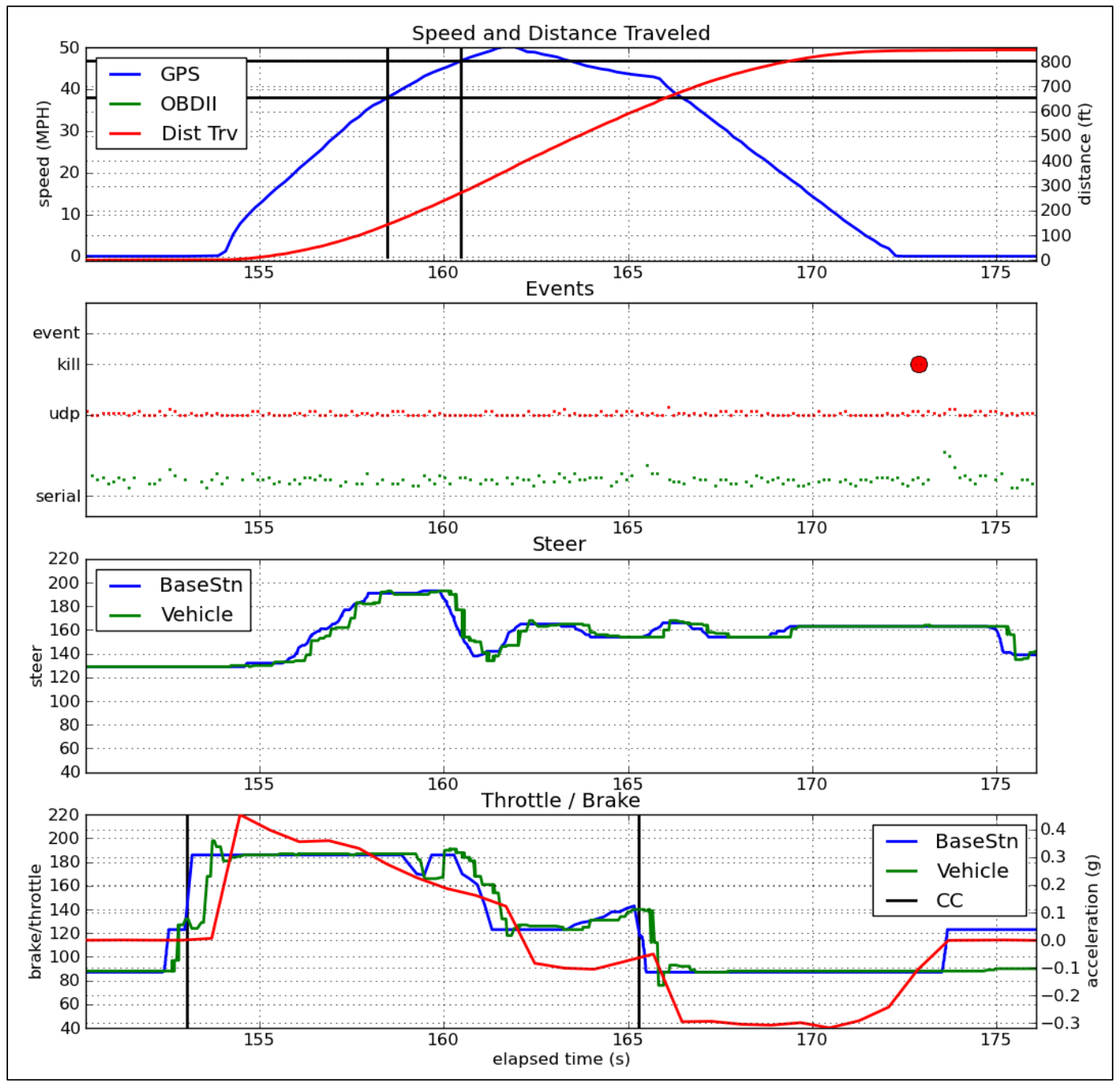

Figure 21 depicts a trial test, not a vehicle crash test. The cruise control was incorrectly tuned, and the vehicle overshot the target maximum speed. The red line on the throttle/brake chart, which indicates the acceleration and deceleration of the vehicle, should have been at zero when the target impact speed was achieved at $t=159$. At $t=165$, the brake was applied by remote pilot, which disengaged the cruise controller. At $t=173$, the kill signal was sent, which shuts off the vehicle engine. Conducting this test allows for the fine-tuning of the cruise control and measure the braking distance. 
Figure 22. Test 9/30/2011.

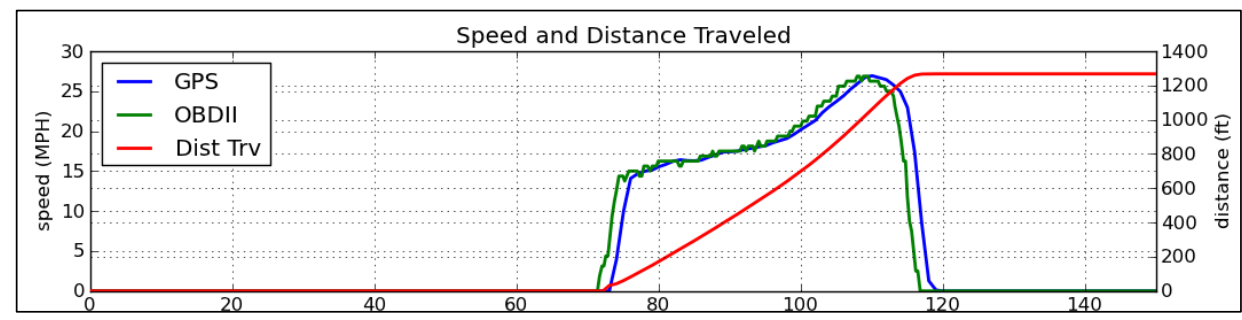

During the test shown in Figure 22, labeled Test 9/30/2011, the on board diagnostics II (OBDII) feature was available on the tested vehicle. The latency depicted in the GPS, the blue line in Figure 22, was determined by the difference in the OBDII reported speed, the green line.

The design team built a test harness to measure the latency of the system steering. The measurement of the latency time for the steering system to achieve the position commanded by the test pilot was less than $0.41 \mathrm{sec}$. This level of latency resulted in a high cognitive load on the test pilot and made training new test pilots difficult. The throttle and brake system latency was never an issue, and there was no requirement to measure the latency.

\subsection{Setup and teardown times}

As the system RPVS evolved through design iterations, the setup and teardown times decreased, as did the number of people required to set up and tear down the system. Maintenance and troubleshooting occurred less frequently and was resolved easily due to less wear and tear on the components. The reduced wear and tear resulted from the system integration into modules.

\subsection{Protocol}

The protocol evolved to ensure the execution of roles and responsibilities in an orderly fashion. Each staff member performed various roles throughout many experiments, and the protocol helped to execute many steps in order to ensure a successfully complete remote driving experiment. Before enforcement of the protocol, there were minor steps missed, such as not setting cameras to record. The protocol performed well as there were no accidents over 15 experiments. 


\subsection{Miscellaneous}

The clearance between the door and control box in vehicle is inadequate because of the possibility of damage to the connectors on the control box when the door is closed. This is a minor design flaw in need of redesign in future versions. Furthermore, a solution is needed for securing the control box to the passenger seat of the vehicle.

During the life cycle of the system, a design requirement for a low latency video signal was recognized. The latency of the video signal was low due to the selection of analog transmitter/receiver. A low latency video signal was required for the test pilot to operate the vehicle.

The original RPVS design called for redundant communication channels to mitigate the risk of complete communication loss. The redundant communications reduced the chances of total communication loss. In more than one instance during experiments, one of the two communication channels failed. However, since there was a redundant channel, there was no instance of total communication loss.

There is a requirement for the vehicle to be in a controlled state, even in the event of communication loss or mechanical failure. Even though communication loss never occurred during an experiment, the fail-safe system always engaged as designed during simulated communication loss. The fail-safe system remained available for engagement in the event of a mechanical failure, such as a jammed throttle. Appendix A lists issues that were encountered and resolved during the development of the RPVS. 


\section{Summary}

This report provides a snapshot of the systems development life cycle for the RPVS, including the requirements, analysis, design, implementation, testing, and maintenance of the system.

Also discussed is the development design of the RPVS and the purpose of each component. Various RPVS and some of the electronic parts are depicted.

The five perspectives of the system are detailed and discussed. They are: (1) the drive system perspective, (2) the instrumentation system, (3) the piloting system, (4) the safety system, and (5) the communication system.

A snapshot of the actions necessary to execute an experiment of the RSVP is also discussed. These actions are (1) the experiment site survey, (2) experiment site setup, (3) rehearsal, (4) test execution, (5) data archiving, (6) reporting.

This report also provided the detailed performance metrics from various tests for the (1) brake and throttle, (2) speed and distance traveled, and (3) acceleration. 


\section{References}

ASTM International. 2015. Standard test method for crash testing of vehicle security barriers. F2656/F2656M-15. West Conshohocken, PA: ASTM International. http://dx.doi.org/10.1520/F2656_F2656M-15

Department of Defense Systems Management College. 2001. Systems engineering fundamentals. Fort Belvoir, VA: Defense Acquisition University Press. Systems Development Life Cycle. http://foldoc.org/Systems\%20Development\%20Life\%20Cycle. Accessed April 14, 2017.

Systems Engineering Overview. http://sebokwiki.org/wiki/Systems_Engineering_Overview. Accessed June 8, 2016. 


\section{Appendix A: Issues}

Following is a list of issues observed and resolved during the development of the RPVS.

- Dash cam video transmission - high latency digital abandoned in favor of analog.

- Foreign-made transmitter/receivers dropped in favor of U.S.-made because Department of Defense (DOD) spectrum management approval application.

- Cruise control added to reduce cognitive load on the test pilot by automating the throttle to achieve target speed.

- A two-stage circuit was integrated that applied push force to engage the brake and then applied a holding force to mitigate overheating in throttle/brake assembly after kill signal and brake application.

- Spotter controls removed since it not used. The idea of the spotter was to enable a role that could insert event markers into log and a second way to send a kill command to the vehicle. 


\section{Appendix B: Materials List}

This is the list of materials used to build the RPVS.

Driver system in vehicle:

- Steel base and frame components

- Turn buckles for adjusting mechanical arms

- Two drill motors

One for controlling brake and accelerator

One for controlling steering

Sony video camera

MHX920A-FT Microhard Systems transmitter and receiver: used for sending/receiving data on serial port

IP2421 Microhard Systems transmitter and receiver: used for sending/receiving data on Ethernet port TX630 Avalon RF Inc. video transmitter FC24008 Laird Technologies Antennae - 3 mounted on roof of test vehicle Inertial switches to shut off vehicle at impact (safety feature)

Control box in vehicle:

- Spinerette

- AX2550 motor controller

- Fail-safe/heartbeat listener

- Fuel/braking fail-safe relays

Remote driver station components

- Gaming steering wheel and brake/accelerator

- Driver monitor

- MHX920A-FT Microhard Systems transmitter and receiver: used for sending/receiving data on serial port

IP2421 Microhard Systems transmitter and receiver: used for sending/receiving data on Ethernet port

- DX602 Avalon RF Inc. video receiver -receives the video signal from the camera mounted in test vehicle

- FC24008 Laird Technologies antennae - 2 (two for video receiver)

- 26T-2400 Andrew Corp. antenna - for IP2421 transceiver 
- Y8966 Laird Technologies antenna - for MHX920A-FT transceiver

- Toughbook to run program

- Displays that show speed, distance to impact, and other GPS information 


\section{Appendix C: Rehearsal Checklist}

1. Complete vehicle setup checklist.

2. Run vehicle at various test speeds.

3. Tune cruise control.

4. Make adjustments to pedal actuators.

5. Test braking distance at target speed.

6. Check communication quality, data and video, throughout entire course. 


\section{Appendix D: Vehicle Setup Checklist}




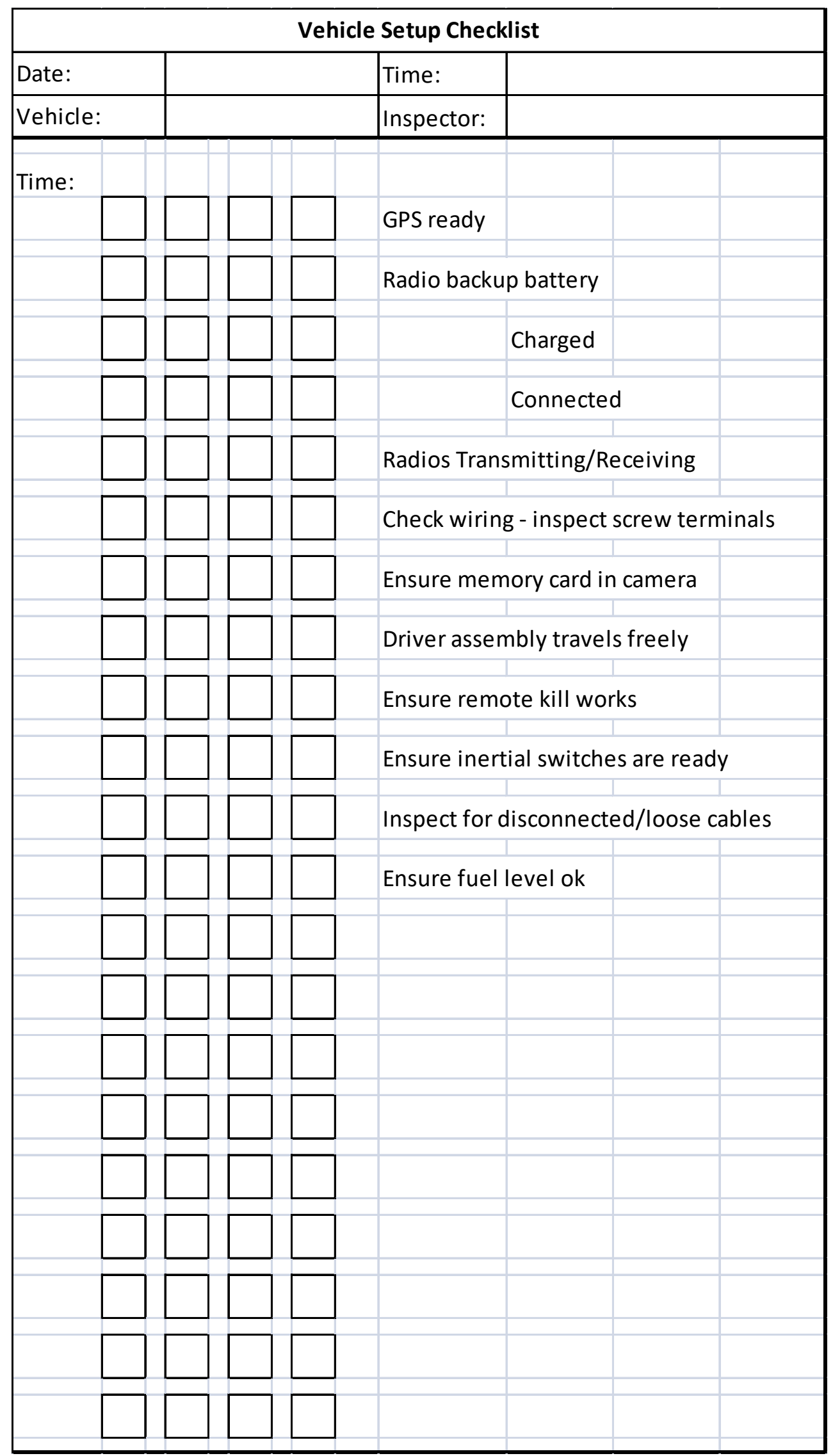




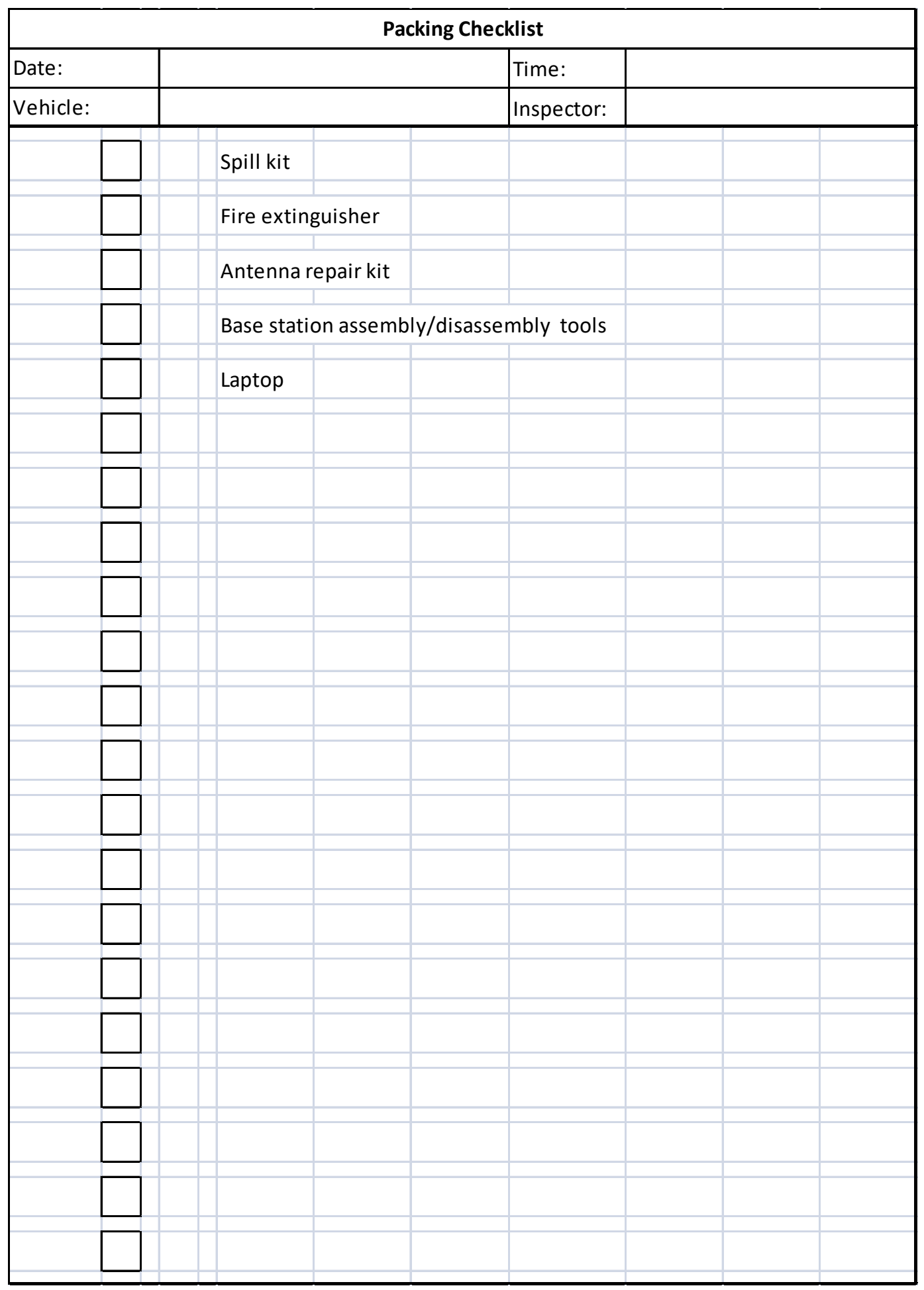




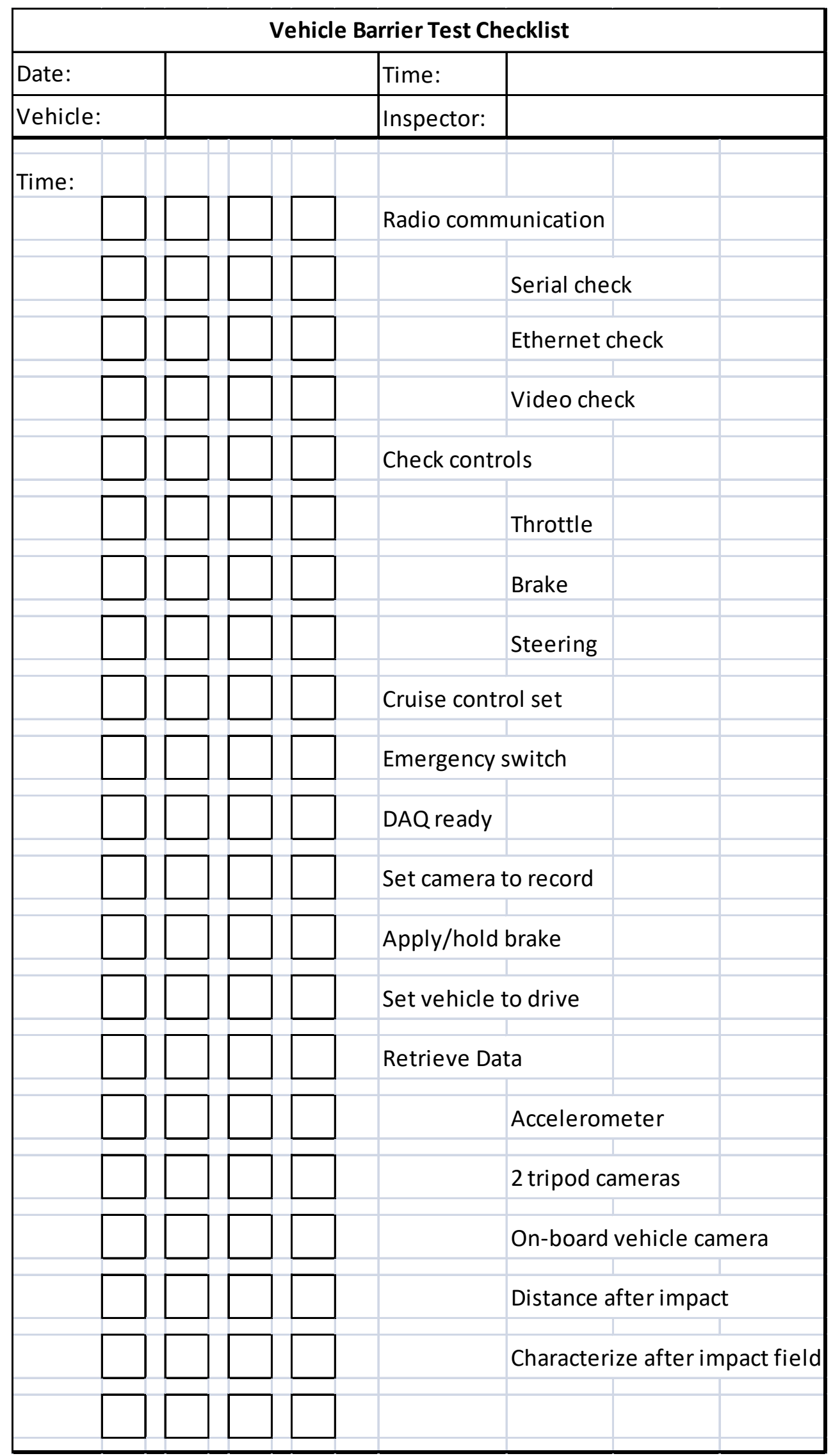




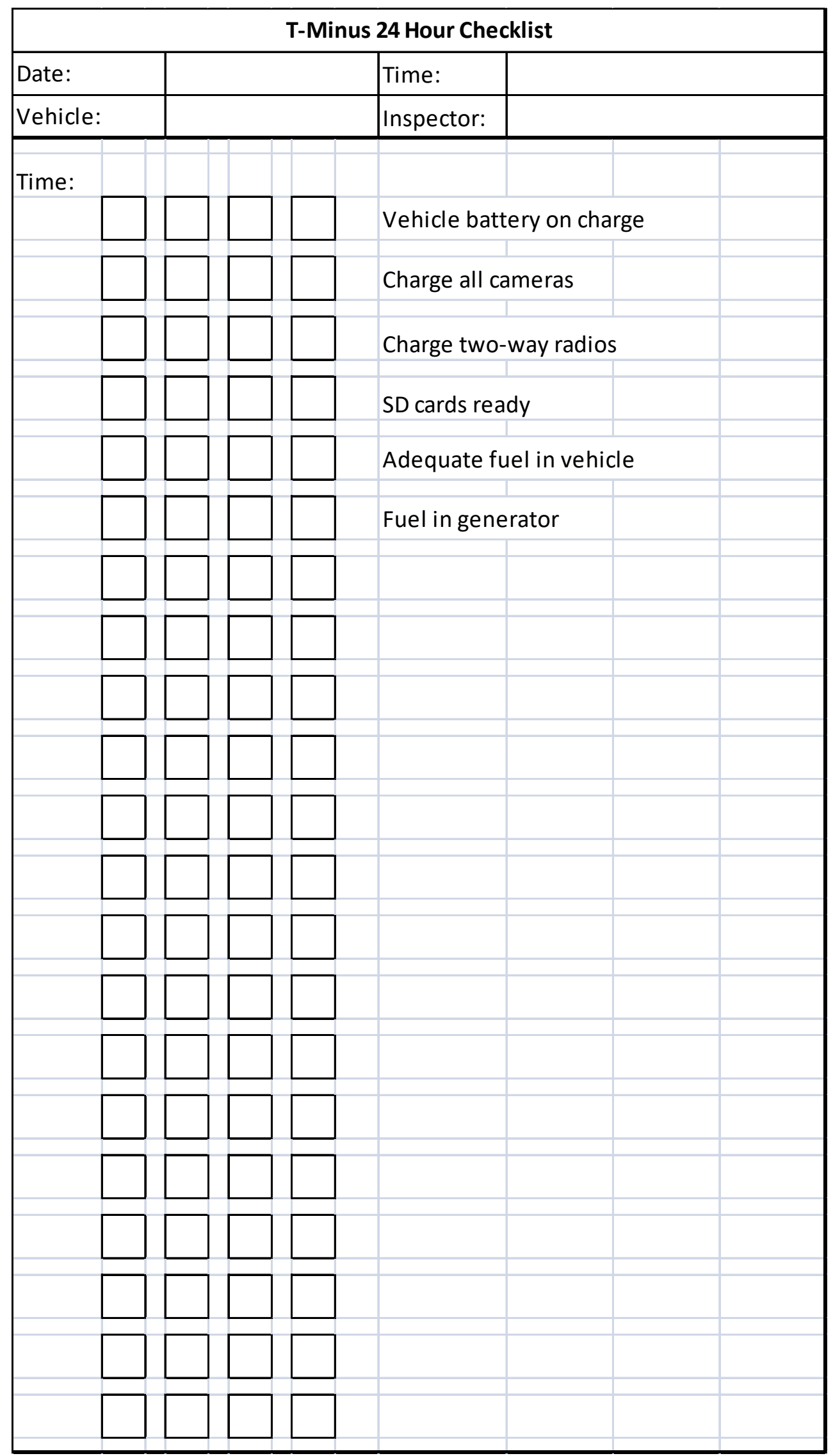




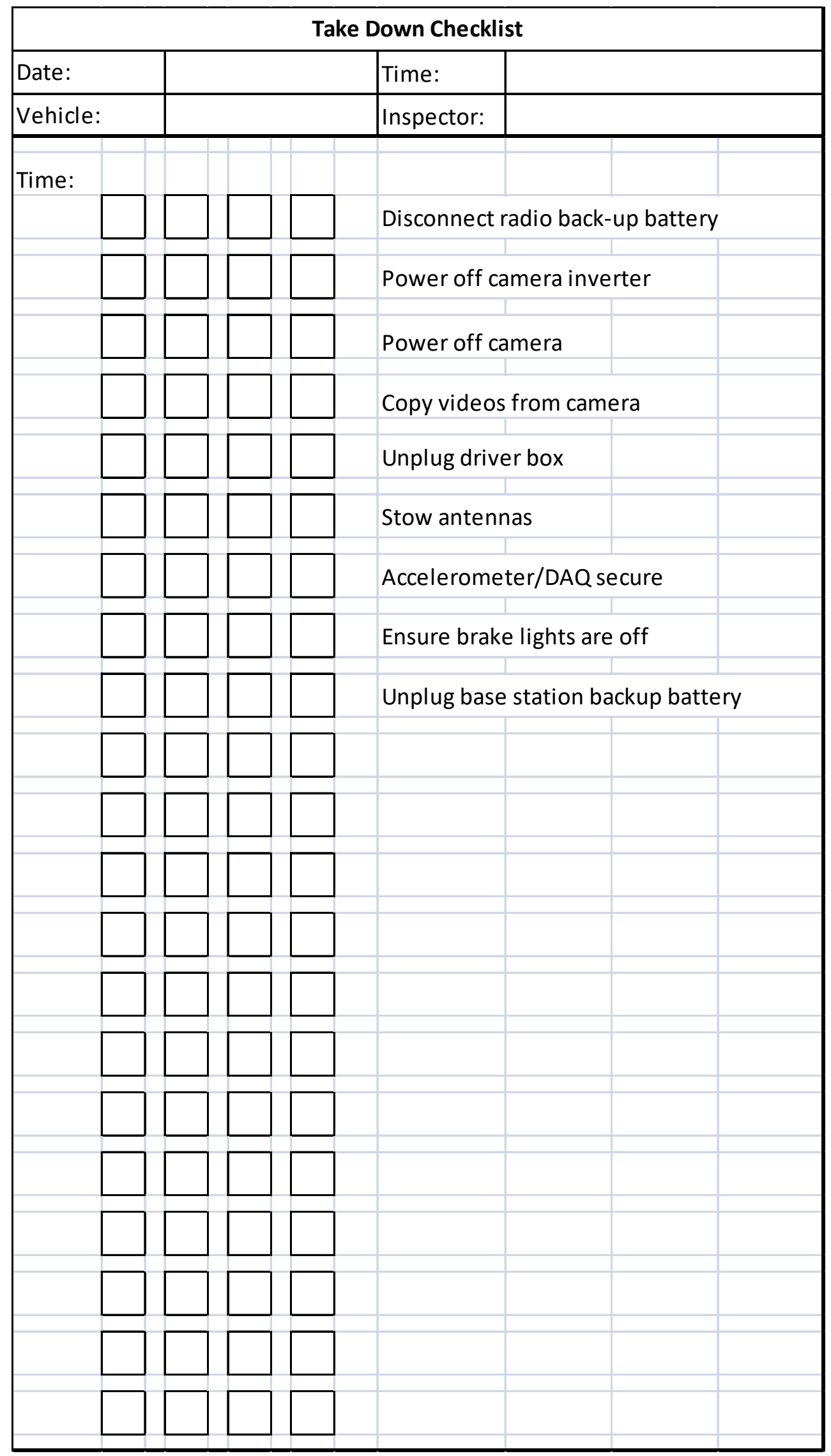




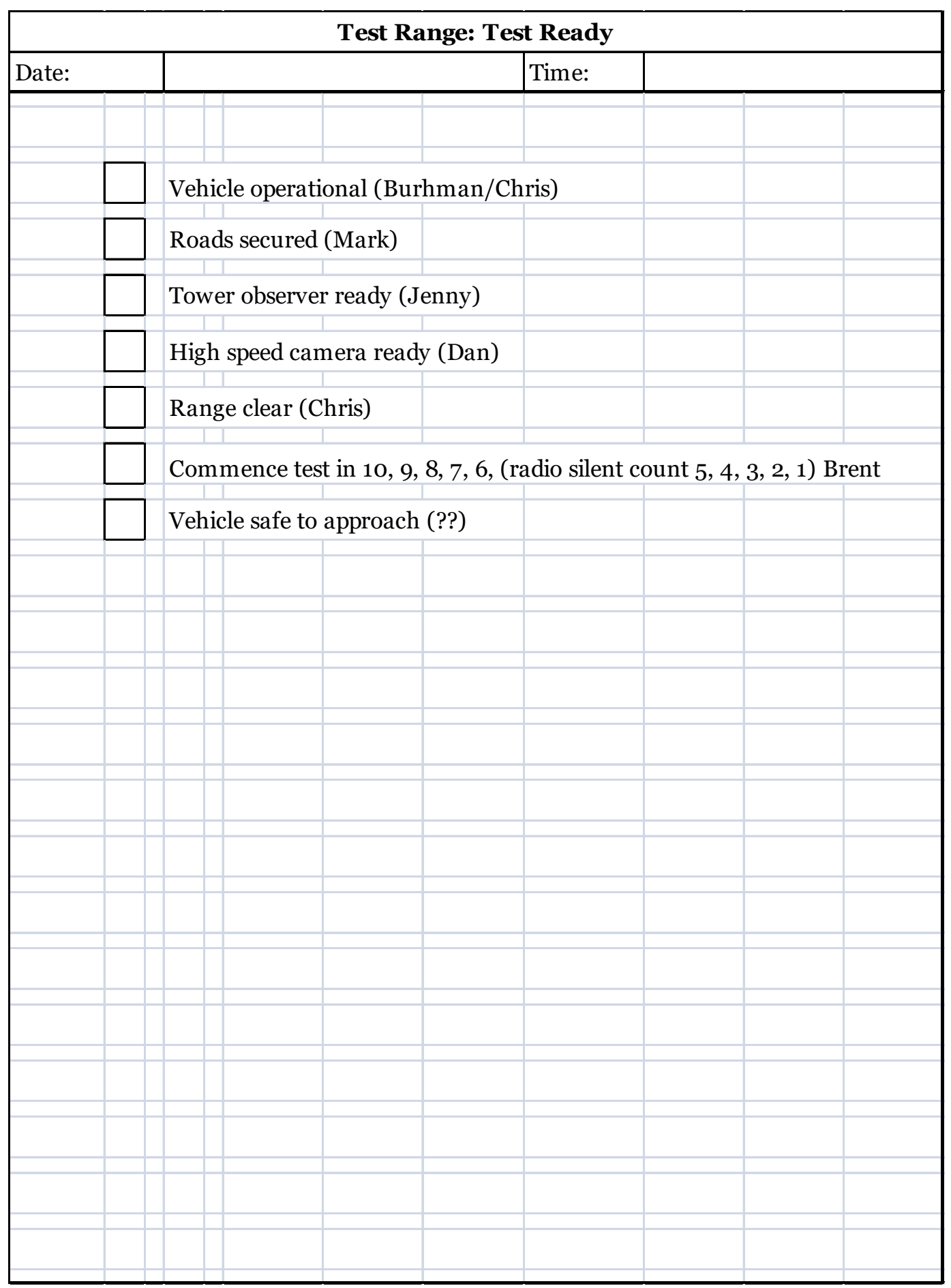




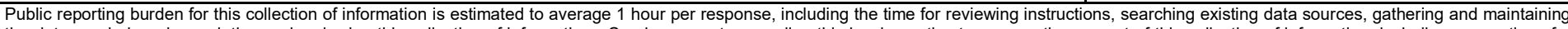

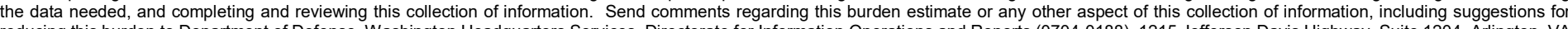

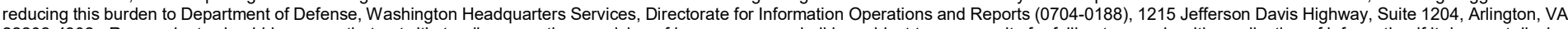

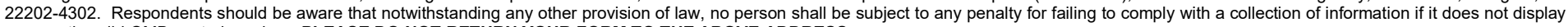
a currently valid OMB control number. PLEASE DO NOT RETURN YOUR FORM TO THE ABOVE ADDRESS.
1. REPORT DATE (DD-MM-YYYY)
2. REPORT TYPE
June 2019
Final report
3. DATES COVERED (From - To)

5a. CONTRACT NUMBER

Developmental Design of the Remotely Piloted Vehicle System

5b. GRANT NUMBER

5c. PROGRAM ELEMENT NUMBER

\section{AUTHOR(S)}

Doris M. Turnage, Brent W. Towne, Burhman Q. Gates, Christopher L. Cummins, Robert D. Ellison, and Clint A. Barela

\section{5d. PROJECT NUMBER}

455651

5e. TASK NUMBER

5f. WORK UNIT NUMBER

\section{PERFORMING ORGANIZATION NAME(S) AND ADDRESS(ES)}

U.S. Army Engineer Research and Development Center

Geotechnical and Structures Laboratory

3909 Halls Ferry Road

Vicksburg, MS 39180-6199

\section{SPONSORING / MONITORING AGENCY NAME(S) AND ADDRESS(ES)}

U.S. Army Corps of Engineers

Washington DC 20314-1000
8. PERFORMING ORGANIZATION REPORT NUMBER

ERDC/GSL TR-19-27

\section{DISTRIBUTION / AVAILABILITY STATEMENT}

Approved for public release; distribution is unlimited.

\section{SUPPLEMENTARY NOTES}

\section{ABSTRACT}

The U. S. Army Engineer Research and Development Center is developing a remotely piloted vehicle driving system for vehicles used in characterizing the performance of barrier systems against vehicle ramming attacks. The design of the driving system allows for expedient installation into a wide range of consumer vehicles with automatic transmissions. Several successful vehicle ramming experiments were conducted using the remotely piloted vehicle ramming system. The design of the experiments allowed the user to characterize the performance of a barrier wall and an entry-point control system. The driver system successfully operated in sedans, trucks, and full-sized vans at target speeds varying from 30 to 60 mph. The vehicles operated safely at distances up to 1,500 feet from the remote base station. The portability of the remotely piloted driver system allows for use at multiple test facilities. This report documents features of the vehicle system and the pilot control system. These features include the vehicle dashboard camera that transmits video to the remote base station, cruise control that operates the vehicle at a target speed, shock sensors that shut down the vehicle within seconds after impact, emergency safety considerations, acceleration and GPS instrumentation measured on vehicle, and portability for incorporation into almost any vehicle.

\begin{tabular}{|ll}
\hline 15. SUBJECT TERMS & Vehicles \\
Obstacles (Military science) & Remotely piloted \\
Roadblocks (Military science) & Remote control
\end{tabular}

16. SECURITY CLASSIFICATION OF:

\begin{tabular}{|l|l|l|}
\hline a. REPORT & b. ABSTRACT & c. THIS PAGE \\
UNCLASSIFIED & UNCLASSIFIED & UNCLASSIFIED \\
\hline
\end{tabular}

17. LIMITATION OF ABSTRACT

SAR
18. NUMBER OF PAGES

59 19a. NAME OF RESPONSIBLE PERSON

19b. TELEPHONE NUMBER (include area code) 